\title{
Fault Tolerant Control of a Proton Exchange Membrane Fuel Cell Using Takagi-Sugeno Virtual Actuators
}

\author{
Damiano Rotondo ${ }^{\mathrm{a}, \mathrm{b}, *}$, Fatiha Nejjari $^{\mathrm{a}}$, Vicenç Puig ${ }^{\mathrm{a}, \mathrm{c}}$ \\ ${ }^{a}$ Department of Automatic Control (ESAII), Technical University of Catalonia (UPC), \\ Rambla de Sant Nebridi 10, 08222 Terrassa (Spain). Tel: +34937398973 \\ ${ }^{b}$ Center for Autonomous Marine Operations and Systems (NTNU-AMOS), Department of \\ Engineering Cybernetics, Norwegian University of Science and Technology, Trondheim, \\ Norway. \\ ${ }^{c}$ Institute of Robotics and Industrial Informatics (IRI), UPC-CSIC, Carrer de Llorens $i$ \\ Artigas, 4-6, 08028 Barcelona (Spain)
}

\begin{abstract}
In this paper, a fault tolerant control (FTC) strategy for proton exchange membrane (PEM) fuel cells based on the use of virtual actuators and the TakagiSugeno (TS) approach is proposed. The overall solution relies on adding a virtual actuator in the control loop to hide the fault from the controller point of view, allowing it to see the same plant as before the fault, such that the stability and some desired performances are preserved. The proposed methodology is based on the use of a reference model, where the resulting nonlinear error model is brought to a Takagi-Sugeno form using a gridding approach. The TS model is suitable for designing a controller using linear matrix inequalities (LMI)-based techniques, such that the resulting closed-loop error system is stable with poles placed in some desired region of the complex plane. Simulation results are used to show the effectiveness of the proposed approach.
\end{abstract}

Keywords: Takagi-Sugeno model, Virtual actuator, Reference model based control, Gain-scheduling, PEM Fuel Cell, LMIs.

\section{Introduction}

Proton exchange membrane (PEM, also known as polymer electrolyte membrane) fuel cells are electrochemical devices which directly convert the chemical energy of hydrogen into electrical energy [1]. Fuel cell systems offer a clean alternative to energy production and are a very active research field because of many possible applications in distributed generation solutions [2]. A good performance of these devices is closely related to the kind of control that is used, so

\footnotetext{
* Corresponding author

Email addresses: damiano.rotondo@yahoo.it (Damiano Rotondo), fatiha.nejjari@upc.edu (Fatiha Nejjari), vicenc.puig@upc.edu (Vicenç Puig)
} 
a study of different control alternatives is considered in [3]. A fuel cell integrates many components into a power system, which supplies electricity to an electric load or to the grid. Several devices, such as DC/DC or DC/AC converters, batteries or ultracapacitors, are included in the system and, in case the fuel cell is not fed directly with hydrogen, a reformer must also be used. Therefore, there are many control loops schemes depending on the devices that must be controlled. The lower control level takes care of the main control loops inside the fuel cell, which are basically fuel/air feeding, humidity, pressure and temperature. The upper control level is in charge of the whole system, integrating the electrical conditioning, storage and reformer (if necessary). Many control strategies have been proposed in the recent literature, e.g. optimal control [4], model predictive control [5] and sliding mode control [6], and several efforts have been put in providing an accurate model for this kind of systems [7, 8].

Since fuel cells are very complex systems, they are vulnerable to faults that can cause their stop or their permanent damage [9, 10]. Some recent works have investigated fault diagnosis [11, 12, 13, 14] and health monitoring [15] strategies for PEM fuel cells, with the aim of obtaining valuable information that could be used to extend the lifetime of the system, thereby avoiding damage in the equipment. Hence, it is interesting to add some fault tolerant capabilities to the control system, in order to maintain the fuel cell operating even in the presence of faults $[16,17]$. Fault tolerant control (FTC) systems are able to maintain desirable closed-loop performance, or with an acceptable degradation, and preserve stability conditions in the presence of component and/or instrument faults $[18,19]$. The existing FTC design techniques can be classified into passive and active approaches (see [20] for a review). Passive FTC techniques exploit the fact that within certain margins, the control law has inherent fault tolerance capabilities, allowing the system to cope with the fault presence, while the $a c$ tive FTC techniques compensate the faults either by selecting a precalculated control law or by synthesizing online a new control strategy. The adaptation of the control law is done using some information about the fault so as to satisfy the control objectives with minimum performance degradation after the fault occurrence.

In recent years, the fault-hiding paradigm has been proposed as an active strategy to obtain fault tolerance [21]. In this paradigm, the controller reconfiguration (CR) unit reconfigures the faulty plant instead of the controller/observer. The nominal controller is kept in the loop by inserting a reconfiguration block between the faulty plant and the nominal controller/observer when a fault occurs. The reconfiguration block is chosen so as to hide the fault from the controller point of view, allowing it to see the same plant as before the fault. The reconfiguration block is named virtual actuator in case of actuator faults and virtual sensor in case of sensor faults. The virtual actuator strategy has been initially proposed in a state space form for LTI systems [22], and successfully extended to linear parameter varying (LPV) [23], Takagi-Sugeno (TS) [24] and piecewise affine [25] systems. An equivalent formulation in input-output form has been proposed in [26].

Recently, the complex and nonlinear dynamics of the power generation sys- 
tems based on fuel cell technology, described in detail in [27], led to the use of linear models that include parameters varying with the operating point (known as LPV models) not only for advanced control techniques [28] but also for modelbased fault diagnosis algorithms [29]. As an alternative to the LPV paradigm, TS systems, as introduced by [30], provide an effective way of representing nonlinear systems with the aid of fuzzy sets, fuzzy rules and a set of local linear models. The overall model is obtained by merging the local models through fuzzy membership functions. In some recent works, the TS paradigm has been successfully applied to the PEM fuel cell system for solving the problems of modeling [31], fault diagnosis [32] and state observation [33].

In this paper, an FTC strategy based on the use of virtual actuators and a TS modeling approach for PEM fuel cells is proposed. The fault tolerant methodology is based on the use of a reference model, where the resulting nonlinear error model is brought to a TS form using a mix of sector nonlinearity and gridding approaches. The TS model is suitable for designing the controller and the virtual actuators, which hide the faults allowing to achieve fault tolerance, using linear matrix inequalities (LMI)-based techniques, such that the resulting closed-loop error system is stable with poles placed in some desired region of the complex plane. Simulation results are used to show the effectiveness of the proposed approach.

The structure of the paper is the following: Section 2 describes the PEM Fuel Cell and presents its nonlinear model. Section 3 shows how, using a reference model, a TS error model, suitable for designing a TS controller using LMI-based techniques, can be obtained. Section 4 presents the proposed FTC strategy based on virtual actuators. The application of the presented theory to a PEM Fuel Cell case study is given in Section 5 and the results obtained in simulation are presented in Section 6. Finally, the main conclusions are outlined in Section 7 .

\section{Description and modeling of PEM fuel cells}

\subsection{PEM fuel cell description}

A fuel cell is an electrochemical energy converter that transforms the chemical energy of fuel into electrical current. It has an electrolyte, a negative electrode and a positive electrode, and it generates direct electrical current through an electrochemical reaction. Typical reactants for fuel cells are hydrogen as fuel and oxygen as oxidant that, once the reaction takes place, produce water and waste heat.

The basic physical structure of a fuel cell consists of an electrolyte layer in contact with a porous anode and cathode electrode plates. There are different kinds of electrolyte layers. Here, a PEM fuel cell is used as a case study. The PEM has a special property: it conducts protons but is impermeable to gas (the electrons are blocked through the membrane). Auxiliary devices are required to ensure the proper operation of the fuel cell stack: an air compressor, a hydrogen tank, a supply manifold and a return manifold. 


\subsection{PEM fuel cell model}

The model used in this work has been presented in [27] and is widely accepted in the control community as a good representation of the behavior of a fuel cell stack (FCS) system. Throughout this work, it is assumed that local loops control the compressor speed $\omega_{c p}$ in order to achieve the desired compressor mass flow $W_{c p}$, and the hydrogen supplied to the anode $m_{H_{2}}$. Hence, the proposed fault tolerant methodology will be applied to the subsystem made up by the supply manifold, the return manifold and the cathode.

The supply manifold includes pipe and stack manifold volumes between the compressor and the fuel cells, and is governed by mass continuity and energy conservation equations [27]:

$$
\begin{aligned}
\dot{m}_{s m} & =W_{c p}-k_{s m, \text { out }}\left[p_{s m}-\frac{\left(m_{O_{2}} R_{O_{2}}+m_{N_{2}} R_{N_{2}}\right) T_{s t}}{V_{c a}}\right] \\
\dot{p}_{s m} & =\frac{\gamma R_{a}}{V_{s m}}\left\{W_{c p}\left[T_{a t m}+\frac{T_{a t m}}{\eta_{c p}}\left(\left(\frac{p_{s m}}{p_{a t m}}\right)^{\frac{\gamma-1}{\gamma}}-1\right)\right]\right. \\
& \left.-k_{\text {sm,out }} T_{s m}\left[p_{s m}-\frac{\left(m_{O_{2}} R_{O_{2}}+m_{N_{2}} R_{N_{2}}\right) T_{s t}}{V_{c a}}\right]\right\}
\end{aligned}
$$

where:

$$
T_{s m}=\frac{p_{s m} V_{s m}}{m_{s m} R_{a}}
$$

The return manifold pressure is governed by mass conservation and the ideal gas law through isothermal assumptions [27]:

$$
\begin{aligned}
\dot{p}_{r m} & =\frac{R_{a} T_{r m}}{V_{r m}} k_{\text {ca,out }}\left(\frac{\left(m_{\mathrm{O}_{2}} R_{\mathrm{O}_{2}}+m_{N_{2}} R_{N_{2}}\right) T_{s t}}{V_{c a}}-p_{r m}\right) \\
& -\frac{R_{a} T_{r m}}{V_{r m}} k_{r m, \text { out }}\left(p_{r m}-p_{a t m}\right)
\end{aligned}
$$
tions:

The cathode flow dynamics is described by the following differential equa-

$$
\begin{gathered}
\dot{m}_{O_{2}}=\chi_{O_{2}, i} k_{s m, \text { out }}\left(p_{s m}-\frac{\left(m_{O_{2}} R_{O_{2}}+m_{N_{2}} R_{N_{2}}\right) T_{s t}}{V_{c a}}\right) \\
-\chi_{O_{2}, o} k_{c a, \text { out }}\left(\frac{\left(m_{O_{2}} R_{O_{2}}+m_{N_{2}} R_{N_{2}}\right) T_{s t}}{V_{c a}}-p_{r m}\right)-\frac{M_{O_{2}} n I_{s t}}{4 F} \\
\dot{m}_{N_{2}}=\left(1-\chi_{O_{2}, i}\right) k_{\text {sm,out }}\left(p_{s m}-\frac{\left(m_{O_{2}} R_{O_{2}}+m_{N_{2}} R_{N_{2}}\right) T_{s t}}{V_{c a}}\right) \\
-\left(1-\chi_{O_{2}, o}\right) k_{\text {ca,out }}\left(\frac{\left(m_{O_{2}} R_{O_{2}}+m_{N_{2}} R_{N_{2}}\right) T_{s t}}{V_{c a}}-p_{r m}\right)
\end{gathered}
$$

with:

$$
\begin{aligned}
\chi_{O_{2}, i} & =\frac{y_{O_{2}, i} M_{O_{2}}}{y_{O_{2}, i} M_{O_{2}}+\left(1-y_{O_{2}, i}\right) M_{N_{2}}} \\
\chi_{O_{2}, o} & =\frac{y_{O_{2}, o} M_{O_{2}}}{y_{O_{2}, o} M_{O_{2}}+\left(1-y_{O_{2}, o}\right) M_{N_{2}}}
\end{aligned}
$$




$$
y_{O_{2}, o}=\frac{m_{O_{2}} R_{O_{2}}}{m_{O_{2}} R_{O_{2}}+m_{N_{2}} R_{N_{2}}}
$$

The model used for control purposes presented in the following has three state variables, i.e. $p_{s m}, p_{r m}$ and $w_{c a}$, defined as:

$$
w_{c a}=m_{O_{2}} R_{O_{2}}+m_{N_{2}} R_{N_{2}}
$$

two control inputs, i.e. $W_{c p}$ and $k_{r m, o u t}$, and an exogenous input, $I_{s t}$, that corresponds to the load, and acts as a disturbance, that can be included in the reference model in order to generate an appropriate feedforward action.

Notice that the following state equation can be obtained from (10), taking into account (5) and (6):

$$
\begin{aligned}
\dot{w}_{c a} & =k_{s m, o u t}\left(p_{s m}-w_{c a} \frac{T_{s t}}{V_{c a}}\right)\left[\chi_{O_{2}, i} R_{O_{2}}+\left(1-\chi_{O_{2}, i}\right) R_{N_{2}}\right] \\
& -k_{c a, \text { out }}\left(w_{c a} \frac{T_{s t}}{V_{c a}}-p_{r m}\right)\left[\chi_{O_{2}, o} R_{O_{2}}+\left(1-\chi_{O_{2}, o}\right) R_{N_{2}}\right]
\end{aligned}
$$

Four sensors are available, measuring $p_{s m}, p_{r m}, T_{s m}$ and the pressure in the cathode $p_{c a}$, related to $w_{c a}$ by:

$$
p_{c a}=w_{c a} \frac{T_{s t}}{V_{c a}}
$$

The efficiency optimization of the current system can be achieved by regulating the oxygen mass inflow towards the stack cathode [34]. If an adequate oxidant flow is ensured through the stack, the load demand is satisfied with minimum fuel consumption. In addition, oxygen starvation and irreversible damage are averted. To accomplish such an oxidant flow is equivalent to maintaining at a suitable value the oxygen stoichiometry, defined as:

$$
\lambda_{O_{2}}=\frac{k_{s m, \text { out }}\left[p_{s m}-\left(m_{O_{2}} R_{O_{2}}+m_{N_{2}} R_{N_{2}}\right) \frac{T_{s t}}{V_{c a}}\right]}{\frac{M_{O_{2}} n I_{s t}}{4 F}}
$$

\section{Model reference control using Takagi-Sugeno techniques}

\subsection{Takagi-Sugeno modeling}

Let us consider that the nonlinear model of the PEM fuel cell can be described by a TS model, which uses a set of local models merged together using fuzzy IF-THEN rules [35], as follows:

$$
\begin{aligned}
& I F \vartheta_{1}(k) \text { is in } M_{i 1} A N D \ldots A N D \vartheta_{p}(k) \text { is in } M_{i p} \\
& \text { THEN } x_{i}(k+1)=A_{i} x(k)+B_{i} u(k) \quad i=1, \ldots, N
\end{aligned}
$$

where $M_{i j}$ denote the fuzzy sets and $N$ is the number of model rules; $x(k) \in \mathbb{R}^{n_{x}}$ is the state vector of the overall system, $x_{i}(k) \in \mathbb{R}^{n_{x}}$ is the state vector of the $i$ th local model, $u(k) \in \mathbb{R}^{n_{u}}$ is the input vector, while $A_{i}$ and $B_{i}$ are matrices 
of appropriate dimensions. $\vartheta_{1}(k), \ldots, \vartheta_{p}(k)$ are premise variables that can be functions of exogenous variables, endogenous variables (states and/or inputs) and/or time. Each linear consequent equation represented by $A_{i} x(k)+B_{i} u(k)$ is called a subsystem.

Given a pair $(x(k), u(k))$, the state of the TS system can easily be inferred by:

$$
x(k+1)=\sum_{i=1}^{N} \rho_{i}(\vartheta(k))\left(A_{i} x(k)+B_{i} u(k)\right)
$$

where $\vartheta(k)=\left[\vartheta_{1}(k), \ldots, \vartheta_{p}(k)\right]^{T}$ is the vector containing the premise variables, and $\rho_{i}(\vartheta(k))$ is defined as follows:

$$
\begin{aligned}
\rho_{i}(\vartheta(k)) & =\frac{w_{i}(\vartheta(k))}{\sum_{i=1}^{N} w_{i}(\vartheta(k))} \\
w_{i}(\vartheta(k)) & =\prod_{j=1}^{p} M_{i j}\left(\vartheta_{j}(k)\right)
\end{aligned}
$$

where $M_{i j}\left(\vartheta_{j}(k)\right)$ is the grade of membership of $\vartheta_{j}(k)$ in $M_{i j}$ and $\rho_{i}(\vartheta(k))$ is such that:

$$
\left\{\begin{array}{l}
\sum_{i=1}^{N} \rho_{i}(\vartheta(k))=1 \\
\rho_{i}(\vartheta(k)) \geq 0, \quad i=1, \ldots, N
\end{array}\right.
$$

\subsection{Takagi-Sugeno reference model and control law}

For the synthesis of the TS controller, the following TS reference model is considered:

$$
\begin{gathered}
\text { IF } \vartheta_{1}(k) \text { is in } M_{i 1} A N D \ldots A N D \vartheta_{p}(k) \text { is in } M_{i p} \\
\text { THEN } x_{r e f, i}(k+1)=A_{i} x_{r e f}(k)+B_{i} u_{r e f}(k) \\
i=1, \ldots, N
\end{gathered}
$$

where $x_{r e f}(k) \in \mathbb{R}^{n_{x}}$ is the reference state vector of the overall system, $x_{r e f, i} \in$ $\mathbb{R}^{n_{x}}$ is the reference state vector of the $i$ th local model, and $u_{r e f}(k) \in \mathbb{R}^{n_{u}}$ is the reference input vector (feedforward action). The reference model generates the trajectory to be tracked by the real system. Given a pair $\left(x_{r e f}(k), u_{r e f}(k)\right)$, the reference state can be inferred as:

$$
x_{r e f}(k+1)=\sum_{i=1}^{N} \rho_{i}(\vartheta(k))\left(A_{i} x_{r e f}(k)+B_{i} u_{r e f}(k)\right)
$$

Thus, considering the error, defined as $e(k) \triangleq x_{r e f}(k)-x(k)$ and the new input $\Delta u(k) \triangleq u_{\text {ref }}(k)-u(k)$ (feedback action), the following TS error system is obtained: 


$$
e(k+1)=\sum_{i=1}^{N} \rho_{i}(\vartheta(k))\left[A_{i} e(k)+B_{i} \Delta u(k)\right]
$$

The error system (21) is controlled through a TS error-feedback controller, defined as follows:

$$
\begin{aligned}
& \text { IF } \vartheta_{1}(k) \text { is in } M_{i 1} A N D \ldots A N D \vartheta_{p}(k) \text { is in } M_{i p} \\
& \text { THEN } \Delta u_{c, i}(k)=K_{i} e(k) \quad i=1, \ldots, N
\end{aligned}
$$

such that the control action to be applied is inferred as the weighted mean:

$$
\Delta u(k)=\sum_{i=1}^{N} \rho_{i}(\vartheta(k)) \Delta u_{c, i}(k)
$$

\subsection{Controller design using an LMI-based approach}

Several techniques, like inverse model control, online adaptive control or model-based predictive control, among others, have been proposed to design controllers for TS systems [36]. In this paper, we consider the LMI-based design approach proposed in [35], where the desired specifications (in this case, stability and pole clustering) are guaranteed using the results from the quadratic Lyapunov framework. This approach has an undeniable advantage in terms of computational complexity, since most of the calculation is performed offline.

In particular, the TS error system (21) with the error-feedback control law (23) is quadratically stable if and only if there exist $X=X^{T}>0$ and $K_{i}$ such that:

$$
\left(\begin{array}{cc}
-X & \left(A_{i}+B_{j} K_{i}\right) X \\
X\left(A_{i}+B_{j} K_{i}\right)^{T} & -X
\end{array}\right)<0
$$

for $i, j=1, \ldots, N$.

On the other hand, pole clustering is based on the results obtained by [37], where subsets $\mathcal{D}$ of the complex plane, referred to as LMI regions, are defined as:

$$
\mathcal{D}=\left\{z \in \mathbb{C}: f_{\mathcal{D}}(z)<0\right\}
$$

where $f_{\mathcal{D}}(z)$ is the characteristic function, defined as:

$$
f_{\mathcal{D}}(z)=\alpha+z \beta+\bar{z} \beta^{T}=\left[\alpha_{h l}+\beta_{h l} z+\beta_{l h} \bar{z}\right]_{h, l \in[1, m]}
$$

where $\alpha=\alpha^{T} \in \mathbb{R}^{m \times m}, \beta \in \mathbb{R}^{m \times m}$ and the notation $\left[\mu_{h l}\right]_{h, l \in[1, m]}$ denotes an $m \times m$ block matrix with generic block $\mu_{k l}$. Hence, the TS error system (21) with error-feedback control law (23) has poles in $\mathcal{D}$ if there exist $X_{\mathcal{D}}=X_{\mathcal{D}}^{T}>0$ and $K_{i}$ such that:

$$
\left[\alpha_{h l} X_{\mathcal{D}}+\beta_{h l}\left(A_{i}+B_{j} K_{i}\right) X_{\mathcal{D}}+\beta_{l h} X_{\mathcal{D}}\left(A_{i}+B_{j} K_{i}\right)^{T}\right] \underset{h, l \in[1, m]}{<0}
$$

for $i, j=1, \ldots, N$. 
Conditions (24)-(27) are bilinear matrix inequalities (BMIs) that can be brought to LMI form by using a single Lyapunov matrix $X=X_{\mathcal{D}}>0$ and through the change of variables $\Gamma_{i} \triangleq K_{i} X$ :

$$
\begin{gathered}
\left(\begin{array}{cc}
-X & A_{i} X+B_{j} \Gamma_{i} \\
\left(A_{i} X+B_{j} \Gamma_{i}\right)^{T} & -X
\end{array}\right)<0 \\
{\left[\alpha_{h l} X+\beta_{h l}\left(A_{i} X+B_{j} \Gamma_{i}\right)+\beta_{l h}\left(A_{i} X+B_{j} \Gamma_{i}\right)^{T}\right]_{h, l \in[1, m]}^{<0}}
\end{gathered}
$$

with $i, j=1, \ldots, N$, that can be solved using available software, e.g. the YALMIP toolbox [38] with SeDuMi solver [39].

Remark 1: In [40], it has been reported that, despite the idea of poles, as introduced, does not have a strict mathematical interpretation in the TS case, it has a strict connection with the dynamical behavior of the system, justifying, from the engineering point of view, the abuse of language. In fact, using LMI regions, it is possible to enforce transient performance specifications, such as decay rates and overshoot suppression (damping).

\section{Fault tolerant control using Takagi-Sugeno virtual actuators}

\subsection{Takagi-Sugeno faulty model}

In this work, two types of actuator faults are considered: multiplicative faults, i.e. changes in the effectiveness of the faulty actuators, and stuck faults, where the inputs delivered by the faulty actuators are blocked to constant values. In the first case, the generic subsystem in (14) becomes as follows:

$$
x_{i}(k+1)=A_{i} x(k)+B_{f, i}(\phi(k)) u(k)
$$

with:

$$
B_{f, i}(\phi(k))=B_{i} \operatorname{diag}\left(\phi_{1}(k), \ldots, \phi_{n_{u}}(k)\right)
$$

where $B_{i}$ denotes the nominal input matrix, and $\left.\left.\phi_{\mu}(k) \in\right] 0,1\right]$ represents the effectiveness of the $\mu$-th actuator, such that the value $\phi_{\mu}=1$ represents the healthy situation.

In the second case, the generic subsystem in (14) becomes:

$$
x_{i}(k+1)=A_{i} x(k)+B_{i}^{*} u(k)+\left(B_{i}-B_{i}^{*}\right) \bar{u}(k)
$$

where $\bar{u}(k)$ is the vector containing the values of the stuck control inputs and $B_{i}^{*}$ is the matrix obtained from $B_{i}$ by replacing the columns corresponding to the stuck actuators with zero vectors.

Then, in the case of multiplicative faults, the reference model subsystems in (19) are changed as follows:

$$
x_{r e f, i}(k+1)=A_{i} x_{r e f}(k)+B_{f, i}(\hat{\phi}(k)) u_{r e f}(k)
$$


where $\hat{\phi}(k)$ is an estimation of the multiplicative actuator faults, while in the case of stuck faults, they become:

$$
x_{r e f, i}(k+1)=A_{i} x_{r e f}(k)+B_{i}^{*} u_{r e f}(k)+\left(B_{i}-B_{i}^{*}\right) \hat{\bar{u}}(k)
$$

where $\hat{\bar{u}}(k)$ is an estimation of the stuck actuator faults.

Then, under the assumption that $\hat{\phi}(k) \cong \phi(k)$ and $\hat{\bar{u}}(k) \cong \bar{u}(k)$ (the case where such an assumption does not hold, i.e. when there is uncertainty in the fault estimation, will be addressed by future research), the generic subsystem of the error model takes the form:

$$
e_{i}(k+1)=A_{i} e(k)+B_{f, i}(\hat{\phi}(k)) \Delta u(k)
$$

or:

$$
e_{i}(k+1)=A_{i} e(k)+B_{i}^{*} \Delta u(k)
$$

for multiplicative or stuck faults, respectively.

Remark 2: The change of the reference model subsystems will also require a modification of the values of the reference inputs $u_{r e f}(k)$ used to generate the desired trajectory.

\subsection{Takagi-Sugeno virtual actuator design}

Here, the concept of virtual actuator introduced in [22] is extended to TS systems. The main idea of this FTC method is to reconfigure the faulty plant such that the nominal controller could still be used without need of retuning it. The plant with the faulty actuators is modified adding the virtual actuator block that masks the fault and allows the controller to see the same plant as before the fault.

In the case of multiplicative faults, the virtual actuator is static and can be expressed as:

$$
\Delta u(k)=\left(\sum_{i=1}^{N} \rho_{i}(\vartheta(k)) B_{f, i}(\hat{\phi}(k))\right)^{\dagger} \sum_{i=1}^{N} \rho_{i}(\vartheta(k)) B_{i} \Delta u_{c, i}(k)
$$

where $\Delta u_{c, i}(k)$ are the outputs of the controller subsystems in (22), and the symbol $\dagger$ denotes the Moore-Penrose pseudoinverse. In this case, the faulthiding property is achieved thanks to the fact that the product:

$$
\left(\sum_{i=1}^{N} \rho_{i}(\vartheta(k)) B_{f, i}(\phi(k))\right)\left(\sum_{i=1}^{N} \rho_{i}(\vartheta(k)) B_{f, i}(\phi(k))\right)^{\dagger}
$$

eliminates the effects of the multiplicative faults.

On the other hand, in the case of stuck faults, the fault tolerance is achieved using the reconfiguration structure expressed by:

$$
\Delta u(k)=\sum_{i=1}^{N} \rho_{i}(\vartheta(k)) \Delta u_{i}(k)
$$


with $\Delta u_{i}(k)$ given by:

$$
\Delta u_{i}(k)=\Delta u_{c, i}(k)-M_{v, i} x_{v}(k)
$$

where the virtual actuator state $x_{v}(k)$ is obtained through the inference:

$$
x_{v}(k)=\sum_{i=1}^{N} \rho_{i}(\vartheta(k)) x_{v, i}(k)
$$

with $x_{v, i}(k)$ provided by the TS virtual actuator subsystems, defined as follows:

$$
\begin{aligned}
& \operatorname{IF} \vartheta_{1}(k) \text { is } M_{i 1} A N D \ldots A N D \vartheta_{p}(k) \text { is } M_{i p} \\
& \text { THEN } x_{v, i}(k+1)=\left(A_{i}+B_{i}^{*} M_{v, i}\right) x_{v, i}(k) \\
& +\left(B_{i}-B_{i}^{*}\right) \Delta u_{c, i}(k) \quad i=1, \ldots, N
\end{aligned}
$$

being $M_{v, i}$ the virtual actuator subsystem gains.

Moreover, in order to achieve the fault-hiding property, the signal entering into the controller is slightly modified, such that the outputs of the controller subsystems in (22) become as follows:

$$
\Delta u_{c, i}(k)=K_{i}\left(e(k)+x_{v}(k)\right)
$$

When the stuck fault appears, the TS virtual actuator reconstructs the vector $\Delta u(k)$ from the outputs of the nominal controller subsystems $\Delta u_{c, i}(k)$, taking into account the fault occurrence. The faulty plant and the TS virtual actuator are called the reconfigured TS plant, which is connected to the nominal TS controller. If the reconfigured TS plant behaves like the nominal plant, the loop consisting of the reconfigured plant and the TS controller behaves like the nominal closed-loop system.

\subsection{Reconfiguration analysis}

In the following, it is shown that thanks to the introduction of the virtual actuator block, the augmented system can be brought to a block-triangular form.

Theorem 1. Consider the augmented system made up by the faulty error system (36), the reconfiguration structure (39)-(40), the virtual actuator (41)-(42) and the control law (43):

$$
\begin{aligned}
& \left(\begin{array}{c}
e(k+1) \\
x_{v}(k+1)
\end{array}\right)=\sum_{i=1}^{N} \rho_{i}(\vartheta(k)) . \\
& \quad\left(\begin{array}{cc}
A_{i}+B_{i}^{*} K_{i} & B_{i}^{*}\left(K_{i}-M_{v, i}\right) \\
\left(B_{i}-B_{i}^{*}\right) K_{i} & A_{i}+B_{i}^{*} M_{v, i}+\left(B_{i}-B_{i}^{*}\right) K_{i}
\end{array}\right)\left(\begin{array}{c}
e(k) \\
x_{v}(k)
\end{array}\right)
\end{aligned}
$$

Then, there exists a similarity transformation such that the state matrix of the augmented system in the new state variables is block-triangular, as follows:

$$
A_{\text {aug }, i}=\left(\begin{array}{cc}
A_{i}+B_{i} K_{i} & 0 \\
\left(B_{i}-B_{i}^{*}\right) K_{i} & A_{i}+B_{i}^{*} M_{v, i}
\end{array}\right)
$$


Proof: The proof is straightforward, and comes from introducing the new state variable $x_{1}(k) \triangleq e(k)+x_{v}(k)$ and considering the state $\left(\begin{array}{lll}x_{1}(k) & x_{v}(k)\end{array}\right)^{T}$.

Looking at (45), it can be seen that the state $x_{1}(k)$ is affected by $K_{i}$ through the matrix $A_{i}+B_{i} K_{i}$, while the state $x_{v}(k)$ is affected by $M_{v, i}$ through the matrix $A_{i}+B_{i}^{*} M_{v, i}$. Hence, the TS controller and the TS virtual actuator can be designed independently.

The design conditions presented in Section 3.3 can be applied to the case of virtual actuator design by making the changes $B_{i} \rightarrow B_{i}^{*}$ and $K_{i} \rightarrow M_{v, i}$.

\subsection{Effects of fault estimation errors}

Due to the presence of noise, disturbances and unmodeled dynamics, the fault estimation is usually affected by errors, such that the ideal assumption that $\hat{\phi}(k) \cong \phi(k)$ and $\hat{\bar{u}}(k) \cong \bar{u}(k)$ does not hold. In this situation, it is important to ensure/enhance the robust stability of the closed-loop system under errors in the fault magnitude estimation [41, 42]. Following the ideas introduced in [43], the more realistic case where $\hat{\phi}(k) \neq \phi(k)$ and $\hat{\bar{u}}(k) \neq \bar{u}(k)$ will be discussed hereafter. By considering $\phi(k)=\hat{\phi}(k)+\Delta \phi(k)$ and $\bar{u}(k)=\hat{\bar{u}}(k)+\Delta \bar{u}(k)$, where $\Delta \phi(k)$ and $\Delta \bar{u}(k)$ are the uncertainties in the estimation of the multiplicative actuator faults and the stuck actuator faults, respectively, and taking into account that:

$$
B_{f, i}(\phi(k))=B_{f, i}(\hat{\phi}(k)+\Delta \phi(k))=B_{f, i}(\hat{\phi}(k))+B_{f, i}(\Delta \phi(k))
$$

where $B_{f, i}(\cdot)$ is defined as in (31), the faulty subsystems (30) and (32) can be rewritten as:

$$
x_{i}(k+1)=A_{i} x(k)+B_{f, i}(\hat{\phi}(k)) u(k)+B_{f, i}(\Delta \phi(k)) u(k)
$$

and:

$$
x_{i}(k+1)=A_{i} x(k)+B_{i}^{*} u(k)+\left(B_{i}-B_{i}^{*}\right) \hat{\bar{u}}(k)+\left(B_{i}-B_{i}^{*}\right) \Delta \bar{u}(k)
$$

respectively. Then, by combining the reference model subsystems (33)-(34) and the faulty subsystems (47)-(48), the generic subsystem of the error model takes the form:

$$
e_{i}(k+1)=A_{i} e(k)+B_{f, i}(\hat{\phi}(k)) \Delta u(k)-B_{f, i}(\Delta \phi(k)) u(k)
$$

and:

$$
e_{i}(k+1)=A_{i} e(k)+B_{i}^{*} \Delta u(k)-\left(B_{i}-B_{i}^{*}\right) \Delta \bar{u}(k)
$$

respectively. It is straightforward to show that the augmented system made up by the faulty error system (49) or (50), the virtual actuator (41)-(42) and the 
control law (43) obeys:

$$
e(k+1)=\sum_{i=1}^{N} \rho_{i}(\vartheta(k))\left[\left(A_{i}+B_{i} K_{i}\right) e(k)-B_{i} \operatorname{diag}(u(k)) \Delta \phi(k)\right]
$$

and:

$$
\begin{aligned}
\left(\begin{array}{c}
x_{1}(k+1) \\
x_{v}(k+1)
\end{array}\right)=\sum_{i=1}^{N} \rho_{i}(\vartheta(k))[ & \left(\begin{array}{cc}
A_{i}+B_{i} K_{i} & 0 \\
\left(B_{i}-B_{i}^{*}\right) K_{i} & A_{i}+B_{i}^{*} M_{v, i}
\end{array}\right)\left(\begin{array}{c}
x_{1}(k) \\
x_{v}(k)
\end{array}\right) \\
& \left.-\left(\begin{array}{c}
\left(B_{i}-B_{i}^{*}\right) \\
0
\end{array}\right) \Delta \bar{u}(k)\right]
\end{aligned}
$$

respectively, where $x_{1}(k) \triangleq e(k)+x_{v}(k)$, and the fact that:

$$
-B_{f, i}(\Delta \phi(k)) u(k)=-B_{i} \operatorname{diag}(\Delta \phi(k)) u(k)=-B_{i} \operatorname{diag}(u(k))(\Delta \phi(k))
$$

has been used.

From (51) and (52), it can be seen that it would be possible to improve the robustness of the FTC system against fault estimation errors using disturbance rejection techniques, such as $\mathcal{H}_{2} / \mathcal{H}_{\infty}$. Details about these techniques can be easily found in the literature, see e.g. [44].

\section{Application to the PEM fuel cell case study}

\subsection{Reference model}

Let us define the following reference model:

$$
\begin{gathered}
\dot{p}_{s m}^{r e f}=\frac{\gamma R_{a}}{V_{s m}}\left\{W_{c p}^{r e f}\left[T_{a t m}+\frac{T_{a t m}}{\eta_{c p}}\left(\left(\frac{\hat{p}_{s m}}{p_{a t m}}\right)^{\frac{\gamma-1}{\gamma}}-1\right)\right]\right. \\
\left.-k_{s m, \text { out }} \hat{T}_{s m}\left[p_{s m}^{r e f}-w_{c a}^{r e f} \frac{T_{s t}}{V_{c a}}\right]\right\} \\
\dot{p}_{r m}^{r e f}=\frac{R_{a} T_{r m}}{V_{r m}} k_{c a, \text { out }}\left(w_{c a}^{r e f} \frac{T_{s t}}{V_{c a}}-p_{r m}^{r e f}\right) \\
-\frac{R_{a} T_{r m}}{V_{r m}} k_{r m, \text { out }}^{r e f}\left(\hat{p}_{r m}-p_{a t m}\right) \\
\dot{w}_{c a}^{r e f}=\chi_{O_{2}, i} k_{s m, \text { out }}\left(p_{s m}^{r e f}-w_{c a}^{r e f} \frac{T_{s t}}{V_{c a}}\right) R_{O_{2}} \\
-\hat{\chi}_{O_{2}, o} k_{c a, o u t}\left(w_{c a}^{r e f} \frac{T_{s t}}{V_{c a}}-p_{r m}^{r e f}\right) R_{O_{2}}-\frac{M_{O_{2}} n I_{s t}}{4 F} \\
+\left(1-\chi_{O_{2}, i}\right) k_{s m, \text { out }}\left(p_{s m}^{r e f}-w_{c a}^{r e f} \frac{T_{s t}}{V_{c a}}\right) R_{N_{2}} \\
-\left(1-\hat{\chi}_{O_{2}, o}\right) k_{c a, o u t}\left(w_{c a}^{r e f} \frac{T_{s t}}{V_{c a}}-p_{r m}^{r e f}\right) R_{N_{2}}
\end{gathered}
$$


where $\hat{p}_{s m}, \hat{p}_{r m}, \hat{T}_{s m}$ and $\hat{\chi}_{O_{2}, o}$ are estimations of $p_{s m}, p_{r m}, T_{s m}$ and $\chi_{O_{2}, o}$, respectively. The available measurements, denoted by $p_{s m}^{y}, p_{r m}^{y}$ and $p_{r m}^{y}$, are filtered to deal with the noise, leading to the following filtered variables:

$$
\begin{aligned}
& \hat{p}_{s m}(k)=\kappa_{p_{s m}} \hat{p}_{s m}(k-1)+\left(1-\kappa_{p_{s m}}\right) p_{s m}^{y}(k) \\
& \hat{p}_{r m}(k)=\kappa_{p_{r m}} \hat{p}_{r m}(k-1)+\left(1-\kappa_{p_{r m}}\right) p_{r m}^{y}(k) \\
& \hat{T}_{s m}(k)=\kappa_{T_{s m}} \hat{T}_{s m}(k-1)+\left(1-\kappa_{T_{s m}}\right) T_{s m}^{y}(k)
\end{aligned}
$$

with the filter parameters $\kappa_{p_{s m}}, \kappa_{p_{r m}}$ and $\kappa_{T_{s m}}$ chosen to be equal to 0.99 .

On the other hand, $\hat{\chi}_{O_{2}, o}$ is obtained as (see (8)-(9)):

$$
\begin{gathered}
\hat{\chi}_{O_{2}, o}=\frac{\hat{y}_{O_{2}, o} M_{O_{2}}}{\hat{y}_{O_{2}, o} M_{O_{2}}+\left(1-\hat{y}_{O_{2}, o}\right) M_{N_{2}}} \\
\hat{y}_{O_{2}, o}=\frac{\hat{m}_{O_{2}} R_{O_{2}}}{\hat{m}_{O_{2}} R_{O_{2}}+\hat{m}_{N_{2}} R_{N_{2}}}
\end{gathered}
$$

where $\hat{m}_{\mathrm{O}_{2}}$ and $\hat{m}_{N_{2}}$ are estimations of $m_{\mathrm{O}_{2}}$ and $m_{N_{2}}$, obtained from steadystate considerations about (5)-(6):

$$
\begin{aligned}
0 & =\chi_{O_{2}, i} k_{s m, \text { out }}\left(\hat{p}_{s m}-\frac{\left(\hat{m}_{O_{2}} R_{O_{2}}+\hat{m}_{N_{2}} R_{N_{2}}\right) T_{s t}}{V_{c a}}\right) \\
- & \hat{\chi}_{O_{2}, o} k_{c a, \text { out }}\left(\frac{\left(\hat{m}_{O_{2}} R_{O_{2}}+\hat{m}_{N_{2}} R_{N_{2}}\right) T_{s t}}{V_{c a}}-\hat{p}_{r m}\right)-\frac{M_{O_{2}} n I_{s t}}{4 F} \\
0 & =\left(1-\chi_{O_{2}, i}\right) k_{s m, o u t}\left(\hat{p}_{s m}-\frac{\left(\hat{m}_{O_{2}} R_{O_{2}}+\hat{m}_{N_{2}} R_{N_{2}}\right) T_{s t}}{V_{c a}}\right) \\
& -\left(1-\hat{\chi}_{O_{2}, o}\right) k_{c a, \text { out }}\left(\frac{\left(\hat{m}_{O_{2}} R_{O_{2}}+\hat{m}_{N_{2}} R_{N_{2}}\right) T_{s t}}{V_{c a}}-\hat{p}_{r m}\right)
\end{aligned}
$$

Given $\hat{p}_{s m}, \hat{p}_{r m}$ and $I_{s t},(60)-(63)$ is a system of two nonlinear equations of variables $\hat{m}_{\mathrm{O}_{2}}$ and $\hat{m}_{N_{2}}$, that can be solved using some appropriate solver, e.g. fsolve in the Matlab Optimization Toolbox [45].

The reference model provides the state trajectory to be tracked by the real PEM fuel cell, starting from the reference inputs $W_{c p}^{r e f}$ and $k_{r m, o u t}^{r e f}$. The values of the reference inputs to be fed to the reference model (feedforward actions) are obtained from steady-state considerations about the fuel cell system, so as to keep the supply manifold pressure and the oxygen stoichiometry at some desired values $p_{s m}^{\infty}$ and $\lambda_{O_{2}}^{r e f}$.

In particular, the following nonlinear equations are obtained:

$$
\begin{gathered}
W_{c p}^{r e f}\left[T_{a t m}+\frac{T_{a t m}}{\eta_{c p}}\left[\left(\frac{\hat{p}_{s m}}{p_{a t m}}\right)^{\frac{\gamma-1}{\gamma}}-1\right]\right] \\
-k_{s m, \text { out }} \hat{T}_{s m}\left(p_{s m}^{\infty}-w_{c a}^{\infty} \frac{T_{s t}}{V_{c a}}\right) T_{s m}=0 \\
k_{c a, \text { out }} w_{c a}^{\infty}\left(\frac{T_{s t}}{V_{c a}}-p_{r m}^{\infty}\right)-k_{r m, \text { out }}^{r e f}\left(\hat{p}_{r m}-p_{a t m}\right)=0
\end{gathered}
$$




$$
\begin{aligned}
& \chi_{O_{2}, i} k_{s m, \text { out }}\left(p_{s m}^{\infty}-w_{c a}^{\infty} \frac{T_{s t}}{V_{c a}}\right) R_{O_{2}} \\
& -\hat{\chi}_{O_{2}, o} k_{c a, \text { out }}\left(w_{c a}^{\infty} \frac{T_{s t}}{V_{c a}}-p_{r m}^{\infty}\right) R_{O_{2}}-\frac{M_{O_{2}} n I_{s t}}{4 F} \\
& +\left(1-\chi_{O_{2}, i}\right) k_{s m, \text { out }}\left(p_{s m}^{\infty}-w_{c a}^{\infty} \frac{T_{s t}}{V_{c a}}\right) R_{N_{2}} \\
& -\left(1-\hat{\chi}_{O_{2}, o}\right) k_{c a, \text { out }}\left(w_{c a}^{\infty} \frac{T_{s t}}{V_{c a}}-p_{r m}^{\infty}\right) R_{N_{2}}=0 \\
& \lambda_{O_{2}}^{r e f}-\frac{\chi_{O_{2}, i} k_{s m, \text { out }}\left(p_{s m}^{\infty}-w_{c a}^{\infty} \frac{T_{s t}}{V_{c a}}\right)}{M_{O_{2}} \frac{n I_{s t}}{4 F}}=0
\end{aligned}
$$

Given $\hat{p}_{s m}, \hat{p}_{r m}, \hat{T}_{s m}, \hat{\chi}_{O_{2}, o}$ and $I_{s t}$, and defined the desired values $p_{s m}^{\infty}$ and $\lambda_{\mathrm{O}_{2}}^{r e f},(64)-(67)$ is a system of four nonlinear equations of variables $W_{c p}^{r e f}$, $k_{r m, o u t}^{r e f}, w_{c a}^{\infty}$ and $p_{r m}^{\infty}$, that can be solved using fsolve [45].

\subsection{Error model}

By subtracting the reference model equations (54)-(56) and the corresponding system equations (2), (4) and (11), and by defining the tracking errors $e_{1} \triangleq p_{s m}^{r e f}-p_{s m}, e_{2} \triangleq p_{r m}^{r e f}-p_{r m}, e_{3} \triangleq w_{c a}^{r e f}-w_{c a}$, and the new inputs $\Delta u_{1} \triangleq W_{c p}^{r e f}-W_{c p}, \Delta u_{2} \triangleq k_{r m, \text { out }}^{r e f}-k_{r m, \text { out }}$, by considering $\hat{T}_{s m} \cong T_{\text {sm }}$, and $\hat{\chi}_{O_{2}, o} \cong \chi_{O_{2}, o}$, the error model for the PEM fuel cell can be brought to the following representation:

$$
\begin{gathered}
\dot{e}_{1}=-\frac{\gamma R_{a}}{V_{s m}} k_{\text {sm }, \text { out }} \hat{T}_{\text {sm }}\left(e_{1}-\frac{T_{s t}}{V_{c a}} e_{3}\right)+b_{11}\left(\hat{p}_{s m}\right) \Delta u_{1} \\
\dot{e}_{2}=-\frac{R_{a} T_{r m}}{V_{r m}} k_{c a, \text { out }}\left(e_{2}-\frac{T_{s t}}{V_{c a}} e_{3}\right)+b_{22}\left(\hat{p}_{r m}\right) \Delta u_{2} \\
\dot{e}_{3}=k_{\text {sm }, \text { out }}\left[\chi_{O_{2}, i} R_{O_{2}}+\left(1-\chi_{O_{2}, i}\right) R_{N_{2}}\right]\left(e_{1}-\frac{T_{s t}}{V_{c a}} e_{3}\right) \\
+k_{c a, \text { out }}\left[\chi_{O_{2}, o} R_{O_{2}}+\left(1-\chi_{O_{2}, o}\right) R_{N_{2}}\right]\left(e_{2}-\frac{T_{s t}}{V_{c a}} e_{3}\right)
\end{gathered}
$$

with:

$$
\begin{gathered}
b_{11}\left(\hat{p}_{s m}\right)=\frac{\gamma R_{a}}{V_{s m}}\left[T_{a t m}+\frac{T_{a t m}}{\eta_{c p}}\left[\left(\frac{\hat{p}_{s m}}{p_{a t m}}\right)^{\frac{\gamma-1}{\gamma}}-1\right]\right] \\
b_{22}\left(\hat{p}_{r m}\right)=-\frac{R_{a} T_{r m}}{V_{r m}}\left(\hat{p}_{r m}-p_{a t m}\right)
\end{gathered}
$$

\subsection{Fault definition}

In this work, three possible faults are considered.

5.3.1. Fault 1: Loss of effectiveness of the compressor $W_{c p}$

By including this fault in the dynamic model of the PEM fuel cell, (2) becomes: 


$$
\begin{aligned}
\dot{p}_{s m} & =\frac{\gamma R_{a}}{V_{s m}} \phi_{c p} W_{c p}\left[T_{a t m}+\frac{T_{a t m}}{\eta_{c p}}\left[\left(\frac{p_{s m}}{p_{a t m}}\right)^{\frac{\gamma-1}{\gamma}}-1\right]\right] \\
& -\frac{\gamma R_{a}}{V_{s m}} k_{s m, \text { out }} T_{s m}\left(p_{s m}-w_{c a} \frac{T_{s t}}{V_{c a}}\right)
\end{aligned}
$$

where $\phi_{c p}$ denotes the multiplicative fault of the air compressor flow.

5.3.2. Fault 2: Loss of effectiveness of the return manifold valve $k_{r m \text {,out }}$

By including this fault, (4) changes to:

$$
\begin{aligned}
\dot{p}_{r m} & =\frac{R_{a} T_{r m}}{V_{r m}} k_{c a, \text { out }}\left(w_{c a} \frac{T_{s t}}{V_{c a}}-p_{r m}\right) \\
& -\frac{R_{a} T_{r m}}{V_{r m}} \phi_{r m} k_{r m, \text { out }}\left(p_{r m}-p_{a t m}\right)
\end{aligned}
$$

where $\phi_{r m}$ denotes the multiplicative fault of the return manifold outlet orifice.

\subsubsection{Fault 3: Stuck of the return manifold valve $k_{r m, \text { out }}$}

In this case, $k_{r m, o u t}$ is stuck to $\bar{k}_{r m, o u t}$, such that (4) becomes:

$$
\begin{aligned}
\dot{p}_{r m} & =\frac{R_{a} T_{r m}}{V_{r m}} k_{c a, \text { out }}\left(w_{c a} \frac{T_{s t}}{V_{c a}}-p_{r m}\right) \\
& -\frac{R_{a} T_{r m}}{V_{r m}} \bar{k}_{r m, \text { out }}\left(p_{r m}-p_{a t m}\right)
\end{aligned}
$$

\subsection{Fault detection/estimation}

In order to apply the proposed strategy, a fault estimation is needed. Hereafter, the fault estimation is formulated as a parameter estimation problem in such a way that any parameter estimation algorithm, such as least squares, could be used. In general, least squares (LS) algorithms can be formulated either in block or in recursive online form [46]. Once the equation is put in regressor form, the recursive formulation [47] and the block formulation [48] are interchangeable.

For estimating the loss of effectiveness of the compressor $\phi_{c p}$, the discretetime version of (73), obtained using an Euler approach with sampling time $T_{s}$, is considered and put in the following regressor form:

$$
z_{s m}(k)=\mu_{s m}(k-1) \phi_{c p}(k-1)
$$

with:

$$
\begin{aligned}
& z_{s m}(k)=p_{s m}(k)-p_{s m}(k-1) \\
& +\frac{\gamma R_{a}}{V_{s m}} k_{s m, o u t} T_{s m}(k-1)\left(p_{s m}(k-1)-w_{c a}(k-1) \frac{T_{s t}}{V_{c a}}\right) T_{s} \\
& \mu_{s m}(k)=\frac{\gamma R_{a}}{V_{s m}} W_{c p}(k)\left\{T_{a t m}+\frac{T_{a t m}}{\eta_{c p}}\left[\left(\frac{p_{s m}(k)}{p_{a t m}}\right)^{\frac{\gamma-1}{\gamma}}-1\right]\right\} T_{s}
\end{aligned}
$$

Similarly, for detecting the presence of a fault in the return manifold valve, the following regressor form is used, that will allow to detect the presence of 
either a loss of effectiveness fault as in (74) or a stuck fault ${ }^{1}$ as in (75):

$$
z_{r m}(k)=\mu_{r m}(k-1) \phi_{r m, o u t}(k-1)
$$

with:

$$
\begin{gathered}
z_{r m}(k)=p_{r m}(k)-p_{r m}(k-1) \\
-\frac{R_{a} T_{r m}}{V_{r m}} k_{c a, \text { out }}\left(w_{c a}(k-1) \frac{T_{s t}}{V_{c a}}-p_{r m}(k-1)\right) T_{s} \\
\mu_{r m}(k)=-\frac{R_{a} T_{r m}}{V_{r m}} k_{r m, \text { out }}(k)\left(p_{r m}(k)-p_{a t m}\right) T_{s}
\end{gathered}
$$

Then, if a block formulation with time window $N_{L S}$ is used, the LS fault estimations are obtained as:

$$
\begin{gathered}
\hat{\phi}_{s m}(k)=M_{s m}(k)^{\dagger} \xi_{s m}(k) \\
\hat{\phi}_{r m}(k)=M_{r m}(k)^{\dagger} \xi_{r m}(k) \\
\xi_{s m}(k)=\left[\begin{array}{c}
z_{s m}(k) \\
z_{s m}(k-1) \\
\vdots \\
z_{s m}\left(k-N_{L S}+1\right)
\end{array}\right] M_{s m}(k)=\left[\begin{array}{c}
\mu_{s m}(k-1) \\
\mu_{s m}(k-2) \\
\vdots \\
z_{r m}(k) \\
\mu_{s m}\left(k-N_{L S}\right)
\end{array}\right] \\
\xi_{r m}(k)=\left[\begin{array}{c}
\mu_{r m}(k-1) \\
\mu_{r m}(k-2) \\
\vdots \\
z_{r m}\left(k-N_{L S}+1\right)
\end{array}\right] M_{r m}(k)=\left[\begin{array}{c}
\mu_{r m}\left(k-N_{L S}\right)
\end{array}\right]
\end{gathered}
$$

with $M_{s m}(k)^{\dagger}$ and $M_{r m}(k)^{\dagger}$ denoting the pseudoinverses of $M_{s m}(k)$ and $M_{r m}(k)$, respectively.

\subsection{Faulty error model of the PEM Fuel Cell}

In the case of loss of effectiveness due to compressor fault, the reference model equation (54) is modified as follows:

$$
\begin{aligned}
\dot{p}_{s m}^{r e f} & =\frac{\gamma R_{a}}{V_{s m}}\left\{\hat{\phi}_{c p} W_{c p}^{r e f}\left[T_{a t m}+\frac{T_{a t m}}{\eta_{c p}}\left(\left(\frac{\hat{p}_{s m}}{p_{a t m}}\right)^{\frac{\gamma-1}{\gamma}}-1\right)\right]\right. \\
& \left.-k_{\text {sm,out }} \hat{T}_{s m}\left[p_{s m}^{r e f}-w_{c a}^{r e f} \frac{T_{s t}}{V_{c a}}\right]\right\}
\end{aligned}
$$

\footnotetext{
${ }^{1}$ Since the effect of the stuck fault will lead to a difference between the real value of the return manifold valve outlet constant, and the control input, a change in $\phi_{r m}$ will appear not only in the case of a loss of effectiveness fault, but in the case of a stuck fault too.
} 
Then, under the assumption that $\hat{\phi}_{c p} \cong \phi_{c p}$, the error equation (68) becomes:

$$
\dot{e}_{1}=-\frac{\gamma R_{a}}{V_{s m}} k_{s m, o u t} \hat{T}_{s m}\left(e_{1}-\frac{T_{s t}}{V_{c a}} e_{3}\right)+b_{11}\left(\hat{p}_{s m}\right) \hat{\phi}_{c p} \Delta u_{1}
$$

Notice that a slight modification of (64) is needed, i.e. replacing $W_{c p}^{r e f}$ with $\hat{\phi}_{c p} W_{c p}^{r e f}$.

On the other hand, when a fault in the return manifold valve is detected, an estimation of the real value of $k_{r m, o u t}$, denoted in the following as $\hat{k}_{r m, \text { out }}$, is obtained using a LS approach similar to the one described in Section 5.4, using the following regressor equation:

$$
z_{r m}(k)=\mu_{k r m}(k-1) k_{r m, o u t}(k-1)
$$

with $z_{r m}(k)$ defined as in (80) and $\mu_{k r m}(k)$ defined as:

$$
\mu_{k r m}(k)=-\frac{R_{a} T_{r m}}{V_{r m}}\left(p_{r m}(k)-p_{a t m}\right) T_{s}
$$

Then, the reference model equation (55) is modified as follows:

$$
\begin{aligned}
\dot{p}_{r m}^{r e f} & =\frac{R_{a} T_{r m}}{V_{r m}} k_{c a, \text { out }}\left(w_{c a}^{r e f} \frac{T_{s t}}{V_{c a}}-p_{r m}^{r e f}\right) \\
& -\frac{R_{a} T_{r m}}{V_{r m}} \hat{k}_{r m, \text { out }}\left(\hat{p}_{r m}-p_{a t m}\right)
\end{aligned}
$$

and the control input $k_{r m, o u t}(k)$ is chosen as $\hat{k}_{r m, o u t} / \hat{\phi}_{r m}$. Then, under the assumption that $\hat{\phi}_{r m} \cong \phi_{r m}$ in the loss of effectiveness fault case, and $\hat{k}_{r m, \text { out }} \cong$ $\bar{k}_{r m, \text { out }}$ in the stuck fault case, in both cases the error equation (69) becomes:

$$
\dot{e}_{2}=-\frac{R_{a} T_{r m}}{V_{r m}} k_{c a, o u t}\left(e_{2}-\frac{T_{s t}}{V_{c a}} e_{3}\right)
$$

Also, (65) should be modified by replacing $k_{r m, o u t}^{r e f}$ with $\hat{k}_{r m, \text { out }}$. Then, the degree of freedom in the choice of $p_{s m}^{\infty}$ is lost, and (64)-(67) become a system of four nonlinear equations of variables $W_{c p}^{r e f}, p_{s m}^{\infty}, w_{c a}^{\infty}$ and $p_{r m}^{\infty}$.

\section{Simulation results}

By considering interval bounds for $p_{s m}$ and $p_{r m}$, i.e. $p_{s m} \in[1.3 \mathrm{~Pa}, 2.3 \mathrm{~Pa}]$ and $p_{r m} \in[1.3 P a, 2.3 P a]$, minimum and maximum values for $b_{11}\left(p_{s m}\right)$ and $b_{22}\left(p_{r m}\right)$ are obtained, as follows: $b_{11} \in\left[6.54 \cdot 10^{6}, 7.96 \cdot 10^{6}\right], b_{22} \in[-2.21$. $\left.10^{12},-0.491 \cdot 10^{12}\right]$. Then, by taking into account the bounds for $T_{s m}$ and $\chi_{O_{2}, o}$, i.e. $T_{s m} \in[250 \mathrm{~K}, 400 \mathrm{~K}]$ and $\chi_{O_{2}, o} \in[0.07,0.21]$, it is possible to use the sector nonlinearity approach [35] for the varying elements of the state matrix obtained from (68)-(70), the gridding approach for $b_{11}\left(p_{s m}\right)$ and $b_{22}\left(p_{r m}\right)$ with $N_{11}=12$ and $N_{22}=12$ points, respectively, and an Euler discretization with sampling 
time $T_{s}=0.01 s$ for transforming (68)-(70) into an equivalent TS model, as follows:

$$
\begin{aligned}
& I F \vartheta_{1}(k) \text { is } M_{i 1} A N D \vartheta_{2}(k) \text { is } M_{i 2} \cdots A N D \vartheta_{4}(k) \text { is } M_{i 4} \\
& T H E N e_{i}(k+1)=A_{i} e(k)+B_{i} \Delta u(k) \quad i=1, \ldots, N
\end{aligned}
$$

where $\vartheta_{1}(k)=\hat{T}_{s m}(k), \vartheta_{2}(k)=\hat{\chi}_{O_{2}, o}(k), \vartheta_{3}(k)=b_{11}\left(p_{s m}(k)\right)$ and $\vartheta_{4}(k)=$ $\left.b_{22}\left(p_{r m}(k)\right)\right)$ are the premise variables, and $N=4 N_{11} N_{22}=576$.

On the other hand, when considering the loss of effectiveness of the compressor (fault 1), the error model becomes:

$$
\begin{aligned}
& I F \vartheta_{1}(k) \text { is } M_{i 1} A N D \vartheta_{2}(k) \text { is } M_{i 2} \cdots A N D \vartheta_{4}(k) \text { is } M_{i 4} \\
& T H E N e_{i}(k+1)=A_{i} e(k)+B_{f, i}\left(\hat{\phi}_{c p}\right) \Delta u(k) i=1, \ldots, N
\end{aligned}
$$

Finally, in the cases of loss of effectiveness of return manifold valve or stuck of return manifold valve (faults 2 and 3, respectively), the error model becomes:

$$
\begin{aligned}
& I F \vartheta_{1}(k) \text { is } M_{i 1} A N D \vartheta_{2}(k) \text { is } M_{i 2} \cdots A N D \vartheta_{4}(k) \text { is } M_{i 4} \\
& T H E N e_{i}(k+1)=A_{i} e(k)+B_{i}^{*} \Delta u(k) \quad i=1, \ldots, N
\end{aligned}
$$

where the matrices $B_{i}^{*}$ are obtained from $B_{i}$ by replacing the second column (the one corresponding to the input $k_{r m, o u t}$ ) with a zero column.

When using a gridding approach to obtain a TS model, as is the case of premise variables $b_{11}\left(p_{s m}\right)$ and $b_{22}\left(p_{s m}\right)$, it is possible to reduce the overall number of LMIs by requiring the stability and desired performance to be strictly guaranteed only at the design points. In this case, as long as the gridding is sufficiently dense, it is reasonable to assume that stability and performance will still hold at operating points different from the design ones. In the case of the PEM fuel cell, this allows to reduce the total number of LMIs to be solved from 331777 to 577 .

In particular, the nominal controller has been designed to assure stability and pole clustering in a circle of radius 0.4 and center $(0.4,0)$. The dynamic virtual actuator that takes into account the faults 2 and 3 has been designed using a circle of radius 0.3 and center $(0.4,0)$.

The results shown in this paper refer to simulations that last $200 \mathrm{~s}$, where the current in the stack $I_{s t}$ (load) and the desired oxygen stoichiometry vary in time, as follows:

$$
I_{s t}(t)=\left\{\begin{array}{lc}
100 & t \leq 40 s \\
70 t-2700 & 40 s<t \leq 41 s \\
170 & 41 s<t \leq 80 s \\
-50 t+4170 & 80 s<t \leq 81 s \\
120 & 81 s<t \leq 120 s \\
40 t-4680 & 120 s<t \leq 121 s \\
160 & 121 s<t \leq 160 s \\
-10 t+1760 & 160 s<t \leq 161 s \\
150 & 161 s<t \leq 200 s
\end{array}\right.
$$




$$
\lambda_{O_{2}}^{r e f}(t)=\left\{\begin{array}{lc}
2 & t \leq 40 s \\
-0.3 t+14 & 40 s<t \leq 41 s \\
1.7 & 41 s<t \leq 80 s \\
0.4 t-30.3 & 80 s<t \leq 81 s \\
2.1 & 81 s<t \leq 120 \mathrm{~s} \\
-0.2 t+26.1 & 120 s<t \leq 121 s \\
1.9 & 121 s<t \leq 160 \mathrm{~s} \\
0.1 t-14.1 & 160 \mathrm{~s}<t \leq 161 \mathrm{~s} \\
2 & 161 \mathrm{~s}<t \leq 200 \mathrm{~s}
\end{array}\right.
$$

and the desired supply manifold pressure is set to $p_{s m}^{\infty}=1.5 \mathrm{~Pa}$.

Real PEM fuel cells are affected by disturbances that can influence the system operation, as well as uncertainties in the model parameters [49]. In fact, the parameters can vary over time because of events such as clogging of air filters and contamination of gas diffusion layers, as mentioned in [50]. Variations in operating conditions, such as temperature, humidity, reactant volumes and compressor parameters, also influence the system parameters [51]. For this reason, it is interesting to perform simulations assuming uncertainty in some parameters.

In addition, the simulations have been performed assuming uncertainty in some parameters, as resumed in Table 1. The nominal values have been taken from [11], and the uncertainty has been generated for each parameter by considering a uniform aleatory distribution around $1 \%$ of the nominal value. The noise in the sensor measurements has been considered as uniformly distributed around $1 \%$ of the measurement. Finally, the dynamic virtual actuator is activated when $\hat{\phi}_{r m}<0.8$. 


\begin{tabular}{|c|c|c|}
\hline Parameter & Nominal value & Real value \\
\hline$\gamma$ & 1.4 & $=$ \\
\hline$\eta_{c p}$ & 0.8 & 0.799 \\
\hline$\chi_{O_{2}, i}$ & 0.233 & $=$ \\
\hline$k_{c a, \text { out }}$ & $2.177 \cdot 10^{-6}$ & $2.170 \cdot 10^{-6}$ \\
\hline$k_{\text {sm, out }}$ & $3.629 \cdot 10^{-6}$ & $3.601 \cdot 10^{-6}$ \\
\hline$n$ & 381 & $=$ \\
\hline$p_{a t m}$ & 101325 & 101040 \\
\hline$y_{O_{2}, i}$ & 0.21 & $=$ \\
\hline$F$ & 96485 & $=$ \\
\hline$M_{N_{2}}$ & $28 \cdot 10^{-3}$ & $=$ \\
\hline$M_{O_{2}}$ & $32 \cdot 10^{-3}$ & $=$ \\
\hline$R_{a}$ & 286.9 & $=$ \\
\hline$R_{N_{2}}$ & 296.8 & $=$ \\
\hline$R_{O_{2}}$ & 259.8 & $=$ \\
\hline$T_{a t m}$ & 298.15 & 296.24 \\
\hline$T_{r m}$ & 300 & 298.69 \\
\hline$T_{s t}$ & 350 & 348.87 \\
\hline$V_{c a}$ & 0.01 & 0.0099 \\
\hline$V_{r m}$ & 0.005 & $=$ \\
\hline$V_{s m}$ & 0.002 & $=$ \\
\hline
\end{tabular}

Table 1: Nominal and real parameter values.

Remark 2: The choice of the oxygen stoichiometry $\lambda_{\mathrm{O}_{2}}^{\text {ref }}$ and the current in the stack $I_{s t}$ is inspired by [28], where the control system is tested against their changes, due to different required net powers. As remarked in several works, e.g. $[52,53]$, the oxygen excess ratio should be regulated around a value of 2 to reduce the formation of stagnant vapor and nitrogen films in the electrochemical area, and prevent the oxygen starvation phenomenon, which has serious consequences on the stack life. In this work, following [28], a non-constant $\lambda_{O_{2}}^{r e f}$ is considered in order to show the capability of the proposed approach to deal with operating point changes. It is also worth noticing that abrupt changes of $I_{s t}(t)$ could be considered as well, even though they would lead to an increase of the oscillatory transients, similarly to the results shown in [28].

Remark 3: Notice that the approach proposed in this paper does not consider the parametric uncertainty during the controller and virtual actuator design. In this sense, the design approach described in Section 4 is not robust, even though the controller and the virtual actuator exhibit some intrinsic robustness due to the presence of a feedback loop. At the expense of increasing the mathematical complexity and computational burden of the design method, it would be possible to incorporate the uncertainties during the design phase using the results proposed in [54]. However, this issue goes beyond the scope of this paper and will be addressed in future work. 


\subsection{Fault scenario 1}

In fault scenario 1 , a loss of effectiveness $\phi_{c p}=0.5$ appearing at time $t=100 \mathrm{~s}$ has been considered. Fig. 1 shows a comparison of the oxygen stoichiometry $\lambda_{O_{2}}$ obtained in both the cases when the nominal controller is used without the proposed FTC strategy (red line) and when the proposed FTC strategy is applied (blue line). The improvement in the tracking performance brought by the virtual actuator can be seen clearly. Fig. 2 shows the estimation of $\phi_{c p}$, demonstrating that the least-squares based algorithm proposed in Section 5.4 is able to estimate correctly the fault (a steady-state offset appears due to the uncertainty in the model used for the simulations). The virtual actuator reconfigures the control input (see blue line in Fig. 3) such that the effect of the control input taking into account the fault (see cyan line in Fig. 3) matches the control input that would be obtained in nominal conditions (see black line in Fig. 3). On the other hand, when no FTC strategy is applied, the intrinsic robustness of the nominal controller against faults due to the feedback action (see red line in Fig. 3) is not sufficient to compensate the fault effect, since there appears a mismatch with respect to the nominal case (see magenta line in Fig. 3). Finally, Fig. 4 shows the estimation of $\chi_{O_{2}, o}$ obtained using the methodology described in Section 5.1. Also in this case, the algorithm succeeds in estimating correctly the oxygen mass fraction in the outlet.

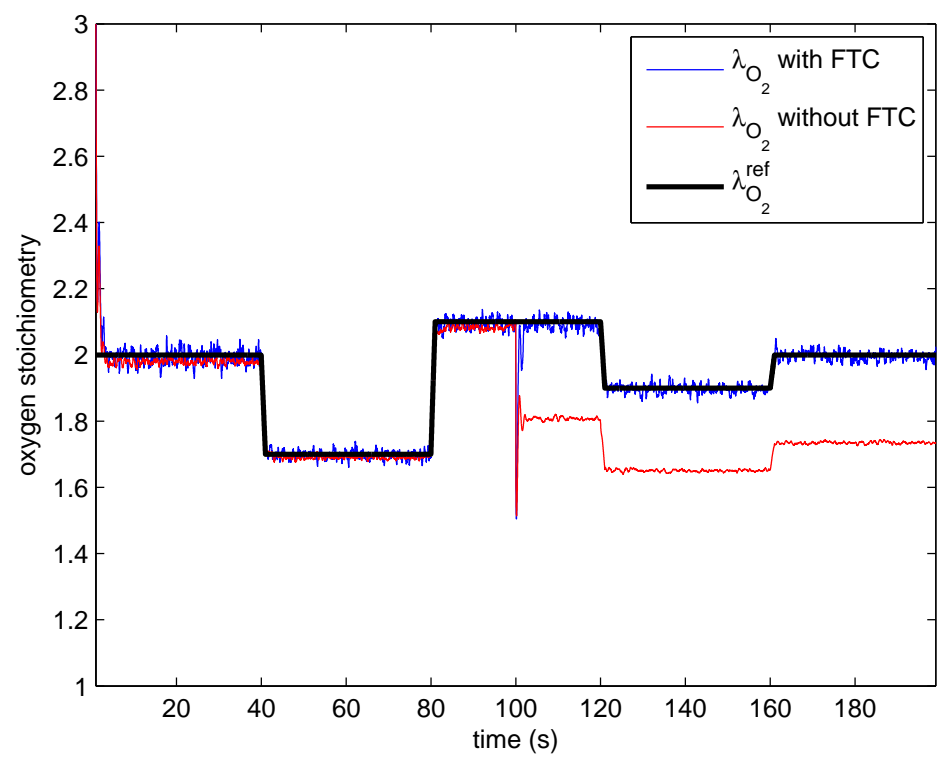

Figure 1: Oxygen stoichiometry $\lambda_{O_{2}}$ in fault scenario 1 . 


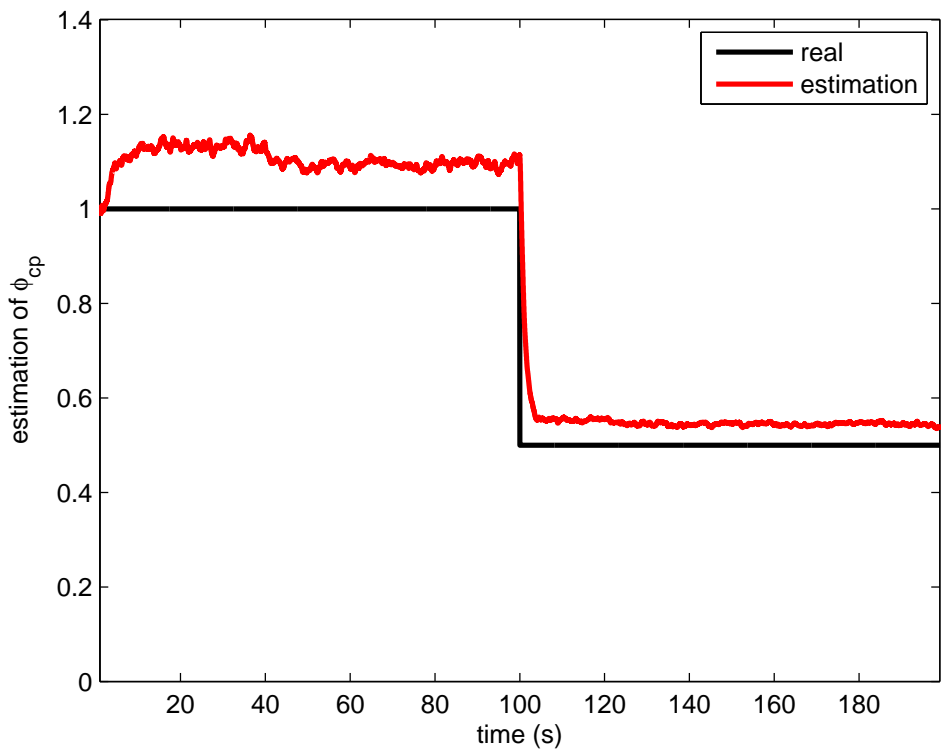

Figure 2: Estimation of $\phi_{c p}$ in fault scenario 1.

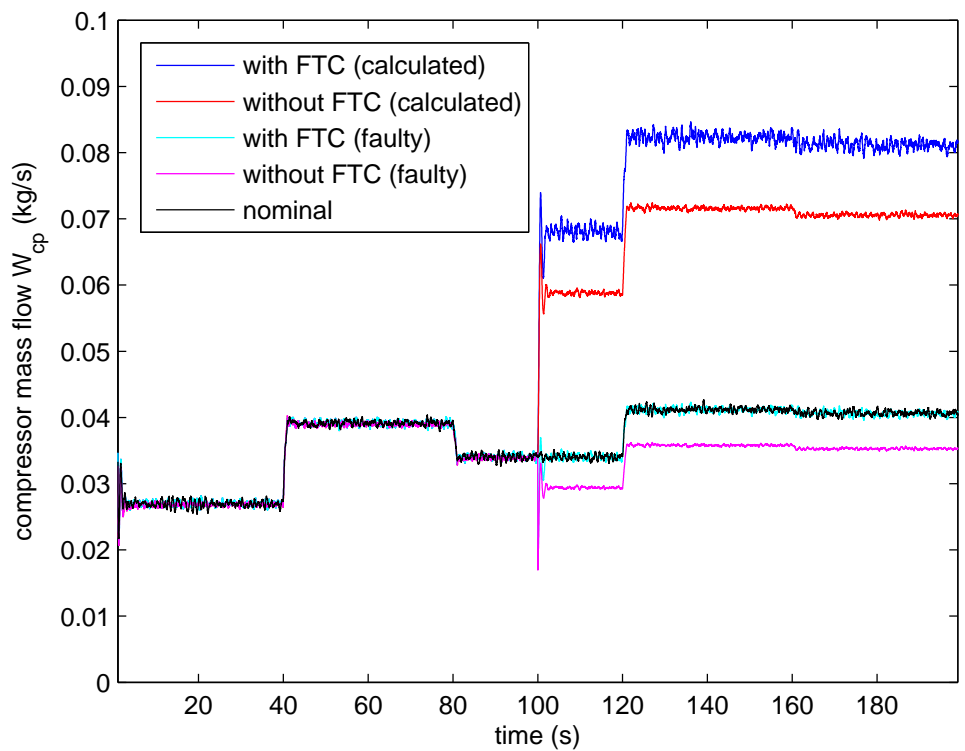

Figure 3: Compressor mass flow $W_{c p}$ in fault scenario 1. 


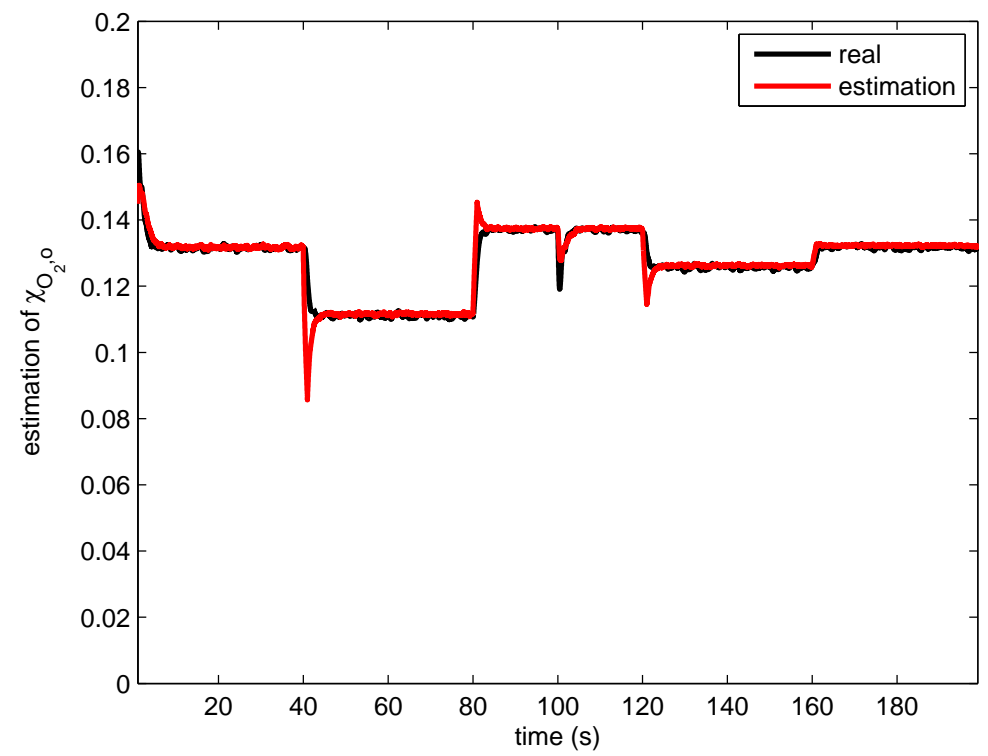

Figure 4: Estimation of $\chi_{\mathrm{O}_{2}, o}$ in fault scenario 1.

\subsection{Fault scenario 2}

In fault scenario 2, a loss of effectiveness $\phi_{r m}=0.5$ appearing at time $t=100 \mathrm{~s}$ has been considered. Similarly to the case of fault scenario 1 , an offset appears in the response of $\lambda_{\mathrm{O}_{2}}$ when no FTC strategy is applied (red line in Fig. $5)$. Despite not being able to reduce the offset to zero, due to the presence of estimation errors caused by the uncertainty, the proposed FTC strategy is able to reduce strongly the effect of the fault on the tracking performance (see blue line in Fig. 5). The dynamic virtual actuator, activated when the estimation of $\phi_{r m}$ goes below 0.8 (see Fig. 6) achieves the fault tolerance by redistributing the controller output on the remaining healthy actuator (the compressor), as shown in Fig. 7. 


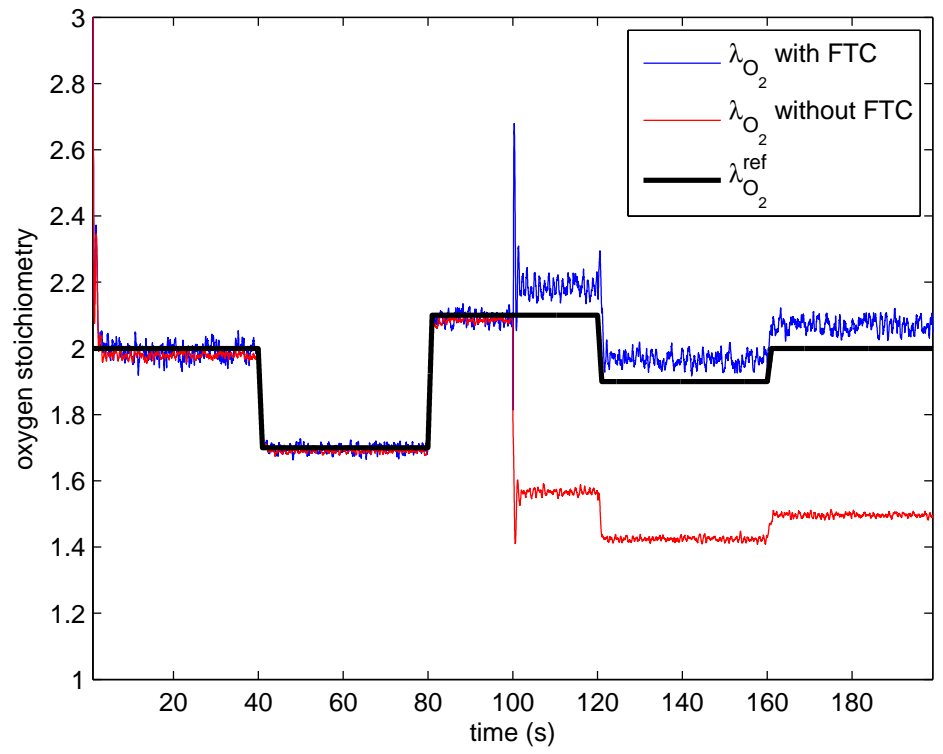

Figure 5: Oxygen stoichiometry $\lambda_{O_{2}}$ in fault scenario 2 .

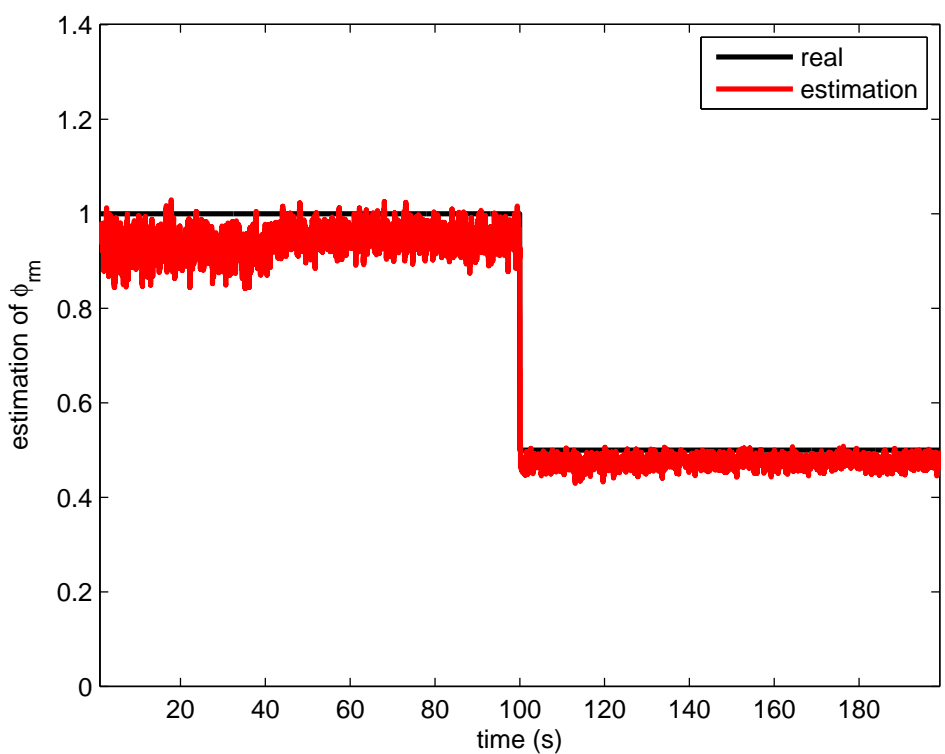

Figure 6: Estimation of $\phi_{r m}$ in fault scenario 2 . 

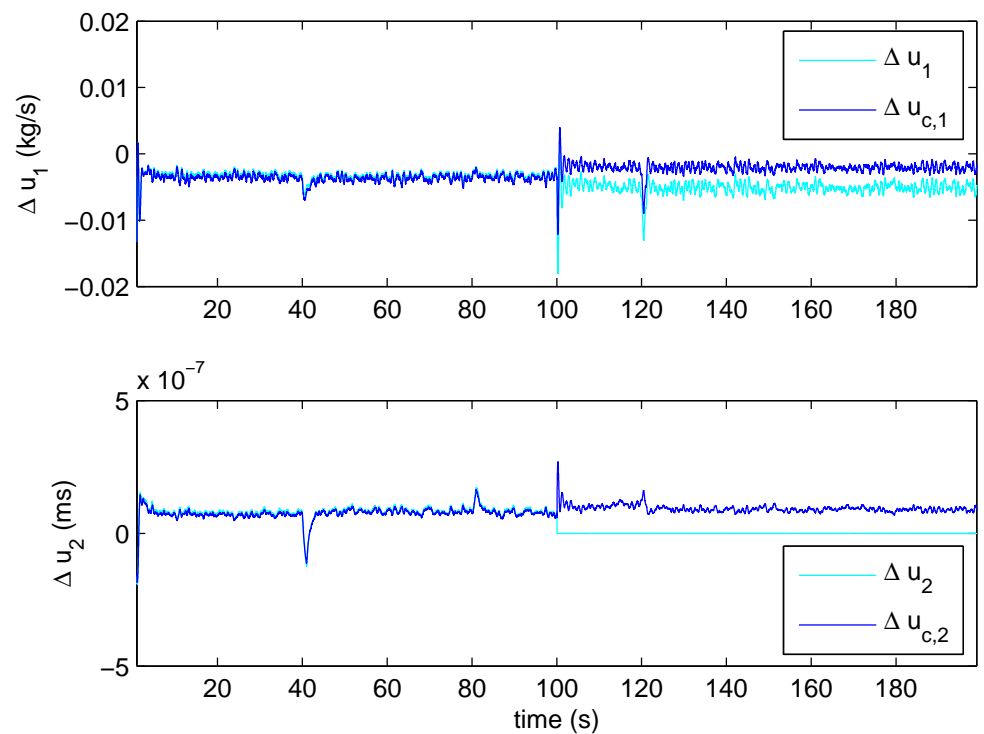

Figure 7: Controller output $\Delta u_{c}$ and reconfiguration brought by the dynamic virtual actuator $\Delta u$ in fault scenario 2 .

\subsection{Fault scenario 3}

In fault scenario 3, the return manifold outlet is stuck starting from time $t=100 \mathrm{~s}$. As shown in Fig. 8, no effect is visible in the oxygen excess ratio

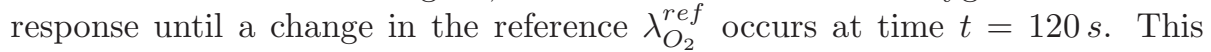
fact is reasonable, because in the interval between the fault occurrence and the reference change, the return manifold outlet is stuck near the correct position that assures an error approximately zero. After the reference change, it can be seen that an offset appears due to the fault (red line in Fig. 8). Also in this case, the reconfiguration brought by the dynamic virtual actuator improves the tracking performance under fault occurrence (blue line in Fig. 8). 


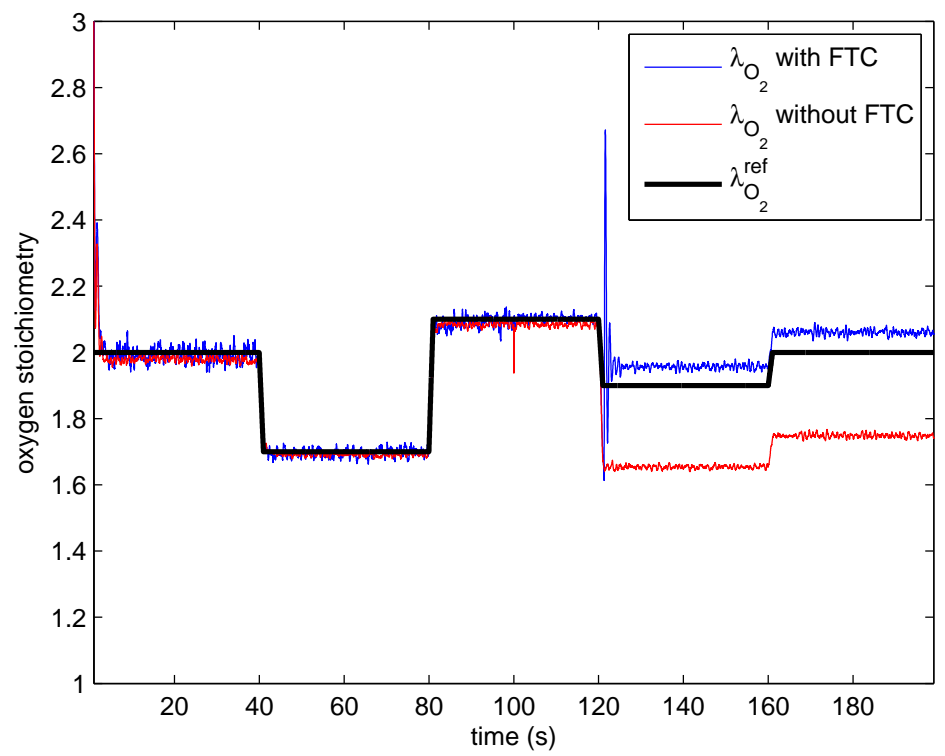

Figure 8: Oxygen stoichiometry $\lambda_{O_{2}}$ in fault scenario 3.

\subsection{Robustness against uncertainties}

In order to assess the robustness of the proposed method against parametric uncertainty and sensor noise, an extensive simulation study has been performed. For each possible uncertainty (noise) magnitude, expressed as the maximum allowed percentage of variation with respect to the values of the nominal parameters (the measured state's real value), five different simulations have been obtained and compared. Table 2 shows the obtained mean errors between the desired and the achieved oxygen stoichiometry, in the case of fault scenario 1. Also, Fig. 9 shows the results corresponding to the worst-case simulations (in terms of biggest mean error), in the cases of uncertainties up to $1 \%$ (blue line), $2 \%$ (red line) and $2.5 \%$ (green line).

\begin{tabular}{|c|c|c|c|c|c|}
\hline Uncertainty & Sim. 1 & Sim. 2 & Sim. 3 & Sim. 4 & Sim. 5 \\
\hline $1 \%$ & 0.0016 & -0.0148 & 0.0084 & -0.0121 & -0.0067 \\
\hline $1.5 \%$ & 0.0149 & -0.0123 & 0.0015 & 0.0199 & -0.0979 \\
\hline $2 \%$ & 0.0306 & 0.0180 & -0.0176 & 0.0093 & -0.0839 \\
\hline $2.5 \%$ & 0.0288 & -0.1748 & 0.0134 & -0.1891 & -0.4407 \\
\hline $3 \%$ & 0.0746 & -0.2908 & -0.3939 & -0.1045 & -0.3485 \\
\hline
\end{tabular}

Table 2: Comparison of results obtained with different uncertainty/noise magnitudes in fault scenario 1 (oxygen stoichiometry mean error). 


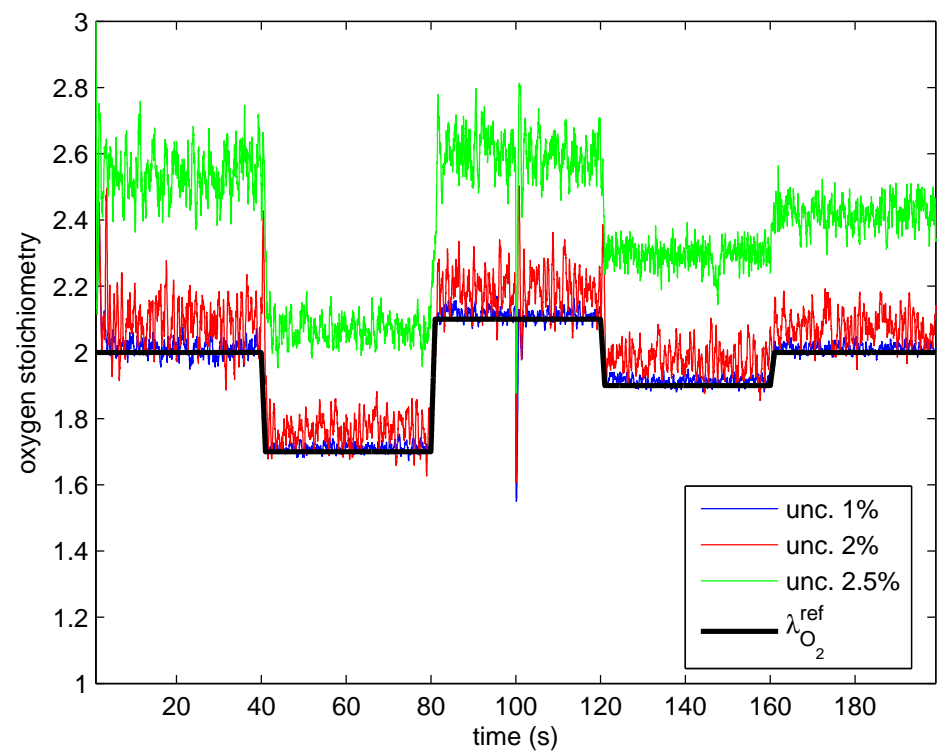

Figure 9: Oxygen stoichiometry $\lambda_{O_{2}}$ in fault scenario 1 (uncertainty).

It can be seen that, although there is an evident degradation of the performances caused by the uncertainty/noise, the results obtained with uncertainty/noise up to $2 \%$ are reasonably acceptable. Similar conclusions have been obtained in the cases of fault scenarios 2 and 3 .

\subsection{Comparison with an LTI fault tolerant control}

Finally, in order to complete the analysis of the proposed approach, the results obtained using the TS model of the PEMFC have been compared with those obtained using an LTI approach. In this case, the LTI model of the PEMFC has been obtained from the LPV one by fixing $p_{s m}=1.8 \mathrm{~Pa}, p_{r m}=$ $1.8 \mathrm{~Pa}, T_{s m}=325 \mathrm{~K}$ and $\chi_{O_{2}}=0.15$. Fig. 10 depicts this comparison (the uncertainty level in the simulation has been fixed to $1 \%$, and the same values for the uncertain parameters have been considered in both the simulations). It can be seen that the TS approach, by taking into account the model variations due to operating point changes, is able to improve the performances in terms of reducing the oxygen stoichiometry tracking error. 


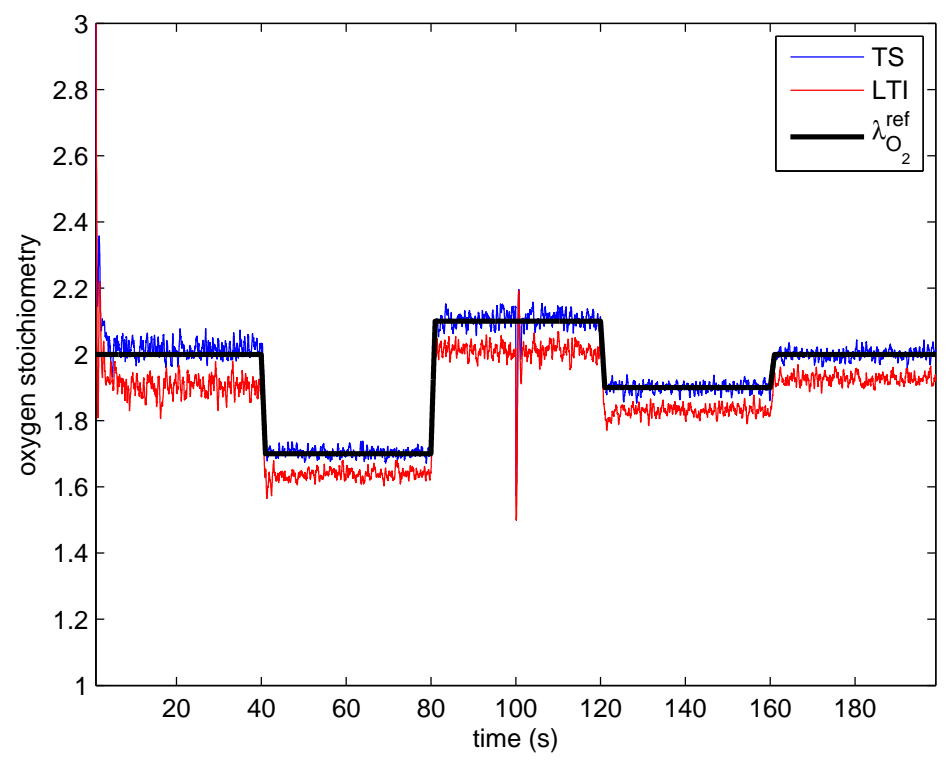

Figure 10: Oxygen stoichiometry $\lambda_{O_{2}}$ in fault scenario 1 (comparison LTI/TS).

\section{Conclusions}

In this paper, an FTC strategy based on the use of virtual actuators in the TS framework for PEM fuel cells has been proposed. The overall solution relies on adding a virtual actuator in the control loop to hide the fault from the controller point of view, allowing it to see the same plant as before the fault, in this way preserving the stability and some desired performances. The proposed methodology is based on the use of a reference model, where the resulting nonlinear error model is brought to a TS form using the gridding approach. The TS model is suitable for designing a controller using LMI-based techniques, such that the resulting closed-loop error system is stable with poles placed in some desired region of the complex plane. Simulation results have shown that if no FTC strategy is applied, undesired offsets would appear. On the other hand, the proposed FTC strategy allows to improve the overall performance in all the considered cases. The comparison with the results obtained using an LTI model of the PEMFC has highlighted the benefits of taking into account the model variations due to operating point changes using the TS paradigm.

In spite of the good results achieved by the proposed approach, there are a few challenging issues that will require further investigation, and that will be considered for future work. For example, in this paper actuator faults were dealt with using the virtual actuator technique. It would be interesting to extend the obtained results to the case of sensor faults, by using the dual technique, i.e. virtual sensors. On the other hand, the proposed strategy will be applied to a real set-up. It is expected that this application will bring more challenges, 
with particular concern to increasing the robustness of the fault tolerant control system against uncertainty, noise and other undesired effects.

\section{Acknowledgements}

This work has been funded by the Spanish Government (MINECO) through the project CICYT ECOCIS (ref. DPI2013-48243-C2-1-R), by MINECO and FEDER through the project CICYT HARCRICS (ref. DPI2014-58104-R), by AGAUR through the contracts FI-DGR 2014 (ref. 2014FI_B1 00172) and FIDGR 2015 (ref. 2015FI_B2 00171) and by the DGR of Generalitat de Catalunya (SAC group Ref. 2014/SGR/374).

\section{Nomenclature}

- $\gamma$ denotes the specific heat capacity of gas

- $\eta_{c p}$ denotes the compressor efficiency

- $\chi_{\mathrm{O}_{2}, i}$ denotes the oxygen mass fraction in the inlet

- $\chi_{\mathrm{O}_{2}, \mathrm{O}}$ denotes the oxygen mass fraction in the outlet

- $k_{c a, \text { out }}$ denotes the cathode outlet flow constant $(\mathrm{ms})$

- $k_{r m, \text { out }}$ denotes the return manifold outlet flow constant $(\mathrm{ms}$ )

- $k_{s m, o u t}$ denotes the supply manifold outlet flow constant $(\mathrm{ms})$

- $m_{N_{2}}$ denotes the mass of nitrogen in the cathode $(\mathrm{kg})$

- $m_{\mathrm{O}_{2}}$ denotes the mass of oxygen in the cathode $(\mathrm{kg})$

- $m_{s m}$ denotes the mass of gas in the supply manifold $(\mathrm{kg})$

- $n$ denotes the number of cells in the FCS

- $p_{a t m}$ denotes the air pressure $(P a)$

- $p_{r m}$ denotes the return manifold pressure $(P a)$

- $p_{s m}$ denotes the supply manifold pressure $(P a)$

- $y_{O_{2}, i}$ denotes the oxygen mole fraction in the inlet

- $y_{\mathrm{O}_{2}, o}$ denotes the oxygen mole fraction in the outlet

- $F$ denotes the Faraday constant $(C / \mathrm{mol})$

- $I_{s t}$ denotes the current in the stack $(A)$

- $M_{N_{2}}$ denotes the nitrogen molar mass $(\mathrm{kg} / \mathrm{mol})$

- $\mathrm{M}_{\mathrm{O}_{2}}$ denotes the oxygen molar mass $(\mathrm{kg} / \mathrm{mol})$

- $R_{a}$ denotes the air gas constant $(\mathrm{J} /(\mathrm{kgK}))$

- $R_{N_{2}}$ denotes the nitrogen gas constant $(J /(k g K))$

- $R_{\mathrm{O}_{2}}$ denotes the oxygen gas constant $(J /(k g K))$

- $T_{a t m}$ denotes the air temperature $(K)$

- $T_{r m}$ denotes the return manifold temperature $(K)$

- $T_{s m}$ denotes the supply manifold temperature $(K)$

- $T_{s t}$ denotes the stack temperature $(K)$

- $V_{c a}$ denotes the cathode volume $\left(m^{3}\right)$

- $V_{r m}$ denotes the return manifold volume $\left(m^{3}\right)$

- $V_{s m}$ denotes the supply manifold volume $\left(\mathrm{m}^{3}\right)$

- $W_{c p}$ denotes the compressor air mass flow rate $(\mathrm{kg} / \mathrm{s})$ 


\section{References}

[1] K.-S. Choi, J. Ahm, J. Lee, N. D. Vinh, H.-M. Kim, K. Park, G. Hwang, An experimental study of scale-up, oxidant, and response characteristics in PEM fuel cells, IEEE Trans. Energy Convers. 29 (2014) 727-734.

[2] F. Gao, B. Blunier, M. G. Simeões, A. Miraoui, PEM fuel cell stack modeling for a real-time emulation in hardware-in-the-loop applications, IEEE Trans. Energy Convers. 26 (2011) 184-194.

[3] J. T. Pukrushpan, A. G. Stefanopoulou, H. Peng, Control of Fuel Cell Power Systems: Principles, Modeling, Analysis and Feedback Design, Series Advances in Industrial Control, Springer, 2004.

[4] A. Niknezhadi, M. Allué-Fantova, C. Kunusch, C. Ocampo-Martínez, Design and implementation of LQR/LQG strategies for oxygen stoichiometry control in PEM fuel cells based systems, J. Power Sources 196 (2011) 4277-4282.

[5] C. Ziogou, S. Papadopoulou, M. C. Georgiadis, S. Voutetakis, On-line nonlinear model predictive control of a PEM fuel cell system, J. Process Contr. 23 (2013) $483-492$.

[6] W. Garcia-Gabin, F. Dorado, C. Bordons, Real-time implementation of a sliding mode controller for air supply on a PEM fuel cell, J. Process Contr. 20 (2010) 325-336.

[7] J. J. Baschuk, X. Li, A general formulation for a mathematical PEM fuel cell model, J. Power Sources 142 (2004) 134-153.

[8] M. Özbek, S. Wang, M. Marx, D. Söffker, Modeling and control of a PEM fuel cell system: a practical study based on experimental defined component behavior, J. Process Contr. 23 (2013) 282-293.

[9] D. Feroldi, Fault diagnosis and fault tolerant control of PEM fuel cell systems, in: M. S. Basualdo, D. Feroldi, R. Outbib (Eds.), PEM fuel cells with bio-ethanol processor systems, Springer, 2012, pp. 185-206.

[10] J. Wu, X. Z. Yuan, J. J. Martin, H. Wang, J. Zhang, J. Shen, S. Wu, W. Merida, A review of PEM fuel cell durability: degradation mechanisms and mitigation strategies, J. Power Sources 184 (2008) 104-119.

[11] A. Aitouche, Q. Yang, B. Ould Bouamama, Fault detection and isolation of PEM fuel cell system based on nonlinear analytical redundancy, Eur. Phys. J.-Appl. Phys. 54 (2011).

[12] C. Damour, M. Benne, B. Grondin-Perez, M. Bessufi, D. Hissel, J. P. Chabriat, Polymer electrolyte membrane fuel cell fault diagnosis based on empirical mode decomposition, Journal of Power Sources 299 (2015) $596-603$. 
[13] P. Polverino, A. Esposito, C. Pianese, B. Ludwig, B. Iwanschitz, A. Mai, On-line experimental validation of a model-based diagnostic algorithm dedicated to a solid oxide fuel cell system, Journal of Power Sources 306 (2016) 646-657.

[14] D. Rotondo, R. M. Fernandez-Canti, S. Tornil-Sin, J. Blesa, V. Puig, Robust fault diagnosis of proton exchange membrane fuel cells using a TakagiSugeno interval observer approach, International Journal of Hydrogen Energy 41 (2016) 2875-2886.

[15] N. Fouquet, C. Doulet, C. Nouillant, G. Dauphin-Tauguy, B. OuldBouamama, Model based PEM fuel cell state-of-health monitoring via ac impedance measurements, Journal of Power Sources 159 (2006) 905-913.

[16] V. Puig, A. Rosich, C. Ocampo-Martínez, R. Sarrate, Fault-tolerant explicit MPC of PEM fuel cells, in: Proc. of the 46th IEEE Conference on Decision and Control, pp. 2657-2662.

[17] F. Bianchi, C. Ocampo-Martínez, C. Kunusch, R. Sanchez-Peña, Faulttolerant unfalsified control for PEM fuel cell systems, IEEE Trans. Energy Convers. 30 (2015) 307-315.

[18] M. Blanke, M. Kinnaert, J. Lunze, M. Staroswiecki, Diagnosis and FaultTolerant Control, Springer-Verlag Berlin Heidelberg, 2006.

[19] H. Noura, D. Theilliol, J. C. Ponsart, A. Chamseddine, Fault-tolerant control systems: Design and practical applications, Springer London, 2009.

[20] Y. Zhang, J. Jiang, Bibliographical review on reconfigurable fault-tolerant control systems, Annu. Rev. Control 32 (2008) 229-252.

[21] T. Steffen, Control reconfiguration of dynamical systems: Linear approaches and structural tests, volume 230 of Lecture Notes in Control and Information Sciences, Springer, 2005.

[22] J. Lunze, T. Steffen, Control reconfiguration after actuator failures using disturbance decoupling methods, IEEE Trans. Autom. Control 51 (2006) 1590-1601.

[23] D. Rotondo, F. Nejjari, V. Puig, A virtual actuator and sensor approach for fault tolerant control of LPV systems, J. Process Contr. 24 (2014) 203-222.

[24] L. Dziekan, M. Witczak, J. Korbicz, Active fault-tolerant control design for Takagi-Sugeno fuzzy systems, Bull. Pol. Acad. Sci. 59 (2011) 93-102.

[25] J. H. Richter, W. P. M. H. Heemels, N. van de Wouw, J. Lunze, Reconfigurable control of piecewise affine systems with actuator and sensor faults: stability and tracking, Automatica 47 (2011) 678-691. 
[26] J. Blesa, D. Rotondo, V. Puig, F. Nejjari, FDI and FTC of wind turbines using the interval observer approach and virtual actuators/sensors, Control Eng. Pract. 24 (2014) 138-155.

[27] J. T. Pukrushpan, H. Peng, A. G. Stefanopoulou, Control-oriented modeling and analysis for automotive fuel cell systems, J. Dyn. Syst.-T. ASME 126 (2004) 14-25.

[28] F. D. Bianchi, C. Kunusch, C. Ocampo-Martínez, R. S. Sánchez-Peña, A gain-scheduled LPV control for oxygen stoichiometry regulation in PEM fuel cell systems, IEEE Trans. Control Syst. Technol. 22 (2014) 1837-1844.

[29] S. de Lira, V. Puig, J. Quevedo, A. Husar, LPV observer design for PEM fuel cell system: Application to fault detection, J. Power Sources 196 (2011) $4298-4305$.

[30] T. Takagi, M. Sugeno, Fuzzy Identification of Systems and Its Applications to Modeling and Control, IEEE Trans. Syst., Man, Cybern. SMC-15 (1985) $116-132$.

[31] S. Olteanu, A. Aitouche, M. Oueidat, A. Jouni, PEM fuel cell modeling and simulation via the Takagi-Sugeno Fuzzy model, in: International Conference on Renewable Energies for Developing Countries (REDEC).

[32] S. Olteanu, A. Aitouche, M. Oueidat, A. Jouni, Fuel cell diagnosis using Takagi-Sugeno observer approach, in: International Conference on Renewable Energies for Developing Countries (REDEC).

[33] S. Olteanu, W. Gauni, A. Aitouche, L. Belkoura, Nonlinear observer for PEM fuel cells using a Takagi-Sugeno approach with unmeasurable premise variables, in: 3rd International Conference on Systems and Control (ICSC), pp. 193-199.

[34] C. Kunusch, P. Puleston, M. Mayosky, Sliding-Mode control of PEM fuel cells, Springer-Verlag, London, U.K., 2012.

[35] K. Tanaka, H. O. Wang, Fuzzy control systems design and analysis: A linear matrix inequality approach, John Wiley and Sons, Inc., 2001.

[36] R. Babuška, Fuzzy modeling for control, Kluwer Academic Publishers, Norwell, MA, USA, 1998.

[37] M. Chilali, P. Gahinet, $H_{\infty}$ design with pole placement constraints: an LMI approach, IEEE Trans. Autom. Control 41 (1996) 358-367.

[38] J. Löfberg, YALMIP: A toolbox for modeling and optimization in MATLAB, in: Proceedings of the CACSD Conference.

[39] J. F. Sturm, Using SeDuMi 1.02, a MATLAB toolbox for optimization over symmetric cones, Optim. method. softw. 11-12 (1999) 625-653. 
[40] P. Korba, R. Babuška, H. Verbruggen, P. Frank, Fuzzy gain scheduling: controller and observer design based on Lyapunov method and convex optimization, IEEE Trans. Fuzzy Syst. 11 (2003) 285-298.

[41] M. M. Seron, J. A. D. Doná, Robust actuator fault compensation accounting for uncertainty in the fault estimation, International Journal of Adaptive Control and Signal Processing 28 (2014) 1440-1453.

[42] B. Jiang, F. N. Chowdhury, Fault estimation and accommodation for linear MIMO discrete-time systems, IEEE Transactions on Control Systems Technology 13 (2015) 493-499.

[43] D. Rotondo, F. Nejjari, V. Puig, J. Blesa, Model reference FTC for LPV systems using virtual actuators and set-membership fault estimation, International Journal of Robust and Nonlinear Control 25 (2015) 735-760.

[44] S.-K. Hong, R. Langari, An LMI-based $\mathcal{H}_{\infty}$ fuzzy control system design with TS framework, Information Sciences 123 (2000) 163-179.

[45] T. Coleman, M. A. Branch, A. Grace, Optimization Toolbox User's Guide, The Mathworks, Inc., Natick, MA, 1999.

[46] J. Jiang, Y. Zhang, A revisit to block and recursive least squares for parameter estimation, Comput. Electr. Eng. 30 (2004) 403-416.

[47] L. Ljung, System Identification: Theory for the user, Prentice Hall, Englewood Cliffs, NJ, USA, 1987.

[48] J. Jiang, Y. Zhang, A novel variable-length sliding window blockwise leastsquares algorithm for on-line estimation of time-varying parameters, Int. J. Adapt. Control 18 (2004) 505-521.

[49] C. Kunusch, P. F. Puleston, M. Mayosky, J. Riera, Sliding mode strategy for PEM fuel cell stacks breathing control using a super twisting algorithm, IEEE Transactions on Control Systems Technology 17 (2009) 167-174.

[50] H. Görgün, M. Arcak, F. Barbir, An algorithm for estimation of membrane water content in PEM fuel cells, Journal of Power Sources 157 (2006) 389394.

[51] I. Matraji, S. Laghrouche, S. Jemei, M. Wack, Robust control of the PEM fuel cell air-feed system via sub-optimal second order sliding mode, Applied Energy 104 (2013) 945-957.

[52] A. Vahidi, A. G. Stefanopoulou, H. Peng, Current management in a hybrid fuel cell power system: A model-predictive control approach, IEEE Trans. Control Syst. Technol. 14 (2006) 1047-1057.

[53] K.-W. Suh, A. G. Stefanopoulou, Performance limitations of air flow control in power-autonomous fuel cell systems, IEEE Trans. Control Syst. Technol. 15 (2007) 465-473. 
[54] D. Rotondo, F. Nejjari, V. Puig, Robust fault tolerant control framework using uncertain Takagi-Sugeno fuzzy models, in: F. Matía, G. N. Marichal, E. Jiménez (Eds.), Fuzzy Modeling and Control: Theory and Applications, Atlantic Press, 2014, pp. 117-133. 